\section{Cahiers de Narratologie}

Analyse et théorie narratives

$40 \mid 2021$

École, Récits, Nation

\title{
Cinématographies éducatives et récits nationaux. Trajectoires comparées de la France et de l'Italie pendant l'entre-deux guerres.
}

\section{Christel Taillibert}

\author{
(2) OpenEdition \\ Journals \\ Édition électronique \\ URL : https://journals.openedition.org/narratologie/12597 \\ DOI : 10.4000/narratologie. 12597 \\ ISSN : 1765-307X \\ Éditeur \\ LIRCES
}

Référence électronique

Christel Taillibert, «Cinématographies éducatives et récits nationaux. Trajectoires comparées de la France et de l'Italie pendant l'entre-deux guerres. ", Cahiers de Narratologie [En ligne], 40 | 2021, mis en ligne le 09 décembre 2021, consulté le 25 décembre 2021. URL : http://journals.openedition.org/ narratologie/12597; DOI : https://doi.org/10.4000/narratologie.12597

Ce document a été généré automatiquement le 25 décembre 2021.

Article L.111-1 du Code de la propriété intellectuelle. 


\title{
Cinématographies éducatives et récits nationaux. Trajectoires comparées de la France et de l'Italie pendant l'entre-deux guerres.
}

\author{
Christel Taillibert
}

\section{Introduction}

1 Envisager l'histoire comparée de l'éducation entre la France et l'Italie dans la première moitié du XX siècle confronte, nécessairement, à la fracture idéologique qui a opposé les deux pays au cours du Ventennio fasciste. L'éducation constitue, en effet, le vecteur par lequel un État cherche à transmettre aux jeunes générations, appelées à constituer les forces vives de demain, un socle identitaire fondateur, un récit partagé, naturel et non contesté. "S'il est un domaine dans lequel les idéologies de la nation sont susceptibles de se heurter de front, c'est bien en éducation ${ }^{1}$ rappelaient, comme une évidence, Angéline Martel et Daniel Villeneuve. L'éducation, telle que nous l'envisageons ici, ne désigne pas la perspective plus individualiste, « conçue avant tout comme une action sur un sujet individuel $»^{2}$, mais plutôt une démarche «s'inscrivant dans une perspective de contrôle, visant à faire acquérir par le sujet apprenant les savoirs prescrits selon des procédures préétablies $»^{3}$, pour reprendre les proposition d'Yves Lenoir et Sabine Vanhulle. Il s'agit donc, dans le cadre d'un État considéré comme un État-Nation, de transmettre par l'éducation un socle commun de valeurs, des modes de socialisation, soit une culture au sens anthropologique du terme, culture partagée qui permet de penser l'Homme, avant tout, comme "le produit d'une éducation ", pour reprendre l'expression de l'anthropologue Jean-Marie Auzias ${ }^{4}$.

2 Parmi les outils auxquels l'appareil éducatif a eu historiquement recours pour assoir cette ambition, le cinématographe a été, dès le début du $\mathrm{XX}^{\mathrm{e}}$ siècle, particulièrement prisé. Ce n'est d'ailleurs pas un hasard s'il a été envisagé comme un outil précieux de 
propagande éducative, dès lors que le développement de ses usages dans un mode documentarisant ${ }^{5}$, a été accompagné d'une croyance en l'irréfutabilité de l'image animée, en sa capacité à " transcrire le réel». Élise Domenach rappelle ainsi ce rôle «mythologique» joué alors par le cinéma, la caméra semblant «révéler automatiquement tout ce qui lui est révélé (...), dépourvue, par son automatisme même, de toute capacité à choisir aussi bien ce qui lui est révélé que ce qu'elle révèle » ${ }^{6}$. Cette confiance absolue en la vérité de l'image animée explique que les premières œuvres de propagande filmée aient été si efficaces, puisque fondées, comme le précise François Niney, sur «le déni de tout point de vue (relatif, subjectif, artiste) au profit de la seule "évidence" : vision sans regard, à prétention universelle, à laquelle le spectateur se rendra sans condition puisqu'elle-même s'affirme "non conditionnée" $»^{7}$.

Posant l'hypothèse que l'histoire de la cinématographie éducative, parce qu'elle été envisagée comme un outil privilégié de propagande, permet de dessiner les contours des idéologies qui l'ont structurée, nous nous proposons dans cet article de poser quelques jalons relatifs à l'histoire comparée de la cinématographie éducative en France et en Italie au cours de la période de l'entre-deux guerres. Nous reviendrons dans un premier temps sur les concepts d'idéologie et de propagande tel que nous les appréhendons dans cette réflexion, avant d'analyser successivement les systèmes qui se sont respectivement mis en place dans la France de la III ${ }^{e}$ République et dans l'Italie fasciste de Benito Mussolini.

\section{Idéologie, propagande et éducation : socle conceptuel}

4 S'intéresser à l'idéologie, entendue à la suite de Teun Van Dijk comme le fondement des représentations sociales partagées par un groupe ${ }^{8}$, implique la prise en considération de trois niveaux d'analyse, qu'Adam Schaff distinguait de la sorte ${ }^{9}$, et que nous retiendrons comme axes d'analyse pertinents pour appréhender l'histoire de la cinématographie éducative : la dimension "génétique ", portant sur les conditions qui ont permis et accompagné l'éclosion d'une idéologie ; la dimension "structurelle ", liée aux élément structurant de l'idéologie, et aux logiques et connaissances qui leur sont associées ; et une dimension "fonctionnelle ", relative aux fonctions de ladite idéologie au sein du groupe où elle est adoptée. Le concept n'est donc pas porteur par essence d'une dimension péjorative ${ }^{10}$, puisque ce partage d'un " discours sur les idées », pour en revenir à l'étymologie du terme ${ }^{11}$, est nécessaire à la structuration d'un groupe social.

Ce « groupe » dont il est question ici pour les chercheurs cités peut prendre des formes très diverses. Dans les deux cas qui nous intéressent, c'est celle d'un État qui fonde et légitime tout à la fois son action politique et son identité sur la base de l'idéologie qui le porte. Cette précision est importante dans la mesure où cette perspective n'implique pas que tous les êtres humains réunis dans le périmètre de cet État partagent forcément les fondements de l'idéologie qui le structure. Des «idéologies dissidentes » peuvent éclore au sein de groupes plus ou moins restreints, et s'exprimer plus ou moins librement en fonction de la liberté d'expression accordée par l'État dont il est question. L'idéologie d'un État se renforce d'ailleurs de ces combats menés contre ces dissidences, par la mise en valeur de l'armature légitimatrice sur la base de laquelle elle a été édifiée. Dans le périmètre historique qui nous occupe, ce phénomène peut être illustré en France par l'idéologie dissidente véhiculée par l'Église catholique, ouvertement opposée aux représentations du monde porteuses de la $\mathrm{III}^{\mathrm{e}}$ République, et 
politiquement associée à des ambitions plus ou moins assumée de restauration de la Monarchie.

6 Si la question de l'idéologie est centrale pour comprendre l'histoire de la cinématographie éducative, c'est que cette dernière s'est vue investie d'un rôle central en termes de propagande, donc en tant que mise en discours de l'idéologie. Le film éducatif a en effet participé à cette «stratégie de communication de masse ayant pour objectifs l'influence de l'opinion et des actions d'individus ou de groupes au moyen d'informations partiales ${ }^{12}$, pour reprendre la définition qu'Étienne Augé proposait en 2007 de la propagande. Comme pour l'idéologie, le concept de propagande n'est pas porteur, en soi, d'un jugement relatif aux valeurs qu'elle véhicule, et ne doit pas être appréhendée au regard des notions toutes relatives de "vrai » et de «faux », même si la sincérité de l'émetteur peut en revanche être légitimement interrogée. C'est ainsi davantage l'intentionnalité de l'action qui est au cœur de ce concept. Aujourd'hui, si le terme a été largement abandonné, tout du moins dans les pays démocratiques, au profit des concepts $d$ ' "information " ou de "communication politique ", il fut très usité pendant l'entre-deux guerres, pour désigner tout à la fois le travail d'endoctrinement des masses opérés par les États totalitaires, et les combats menés dans les démocraties en faveur de l'hygiène, de la prophylaxie, de la modernisation des pratiques agricoles et industrielles, etc.

7 Comme nous l'affirmions précédemment, la cinématographie éducative a été intimement associée à cette œuvre de propagande, et ce dans les deux pays qui nous intéressent. En Italie, elle a accompagné la volonté de rallier les foules, et en particulier la jeunesse, à la pensée fasciste, adhésion envisagée comme un préalable à son esprit de soumission. Et en France, elle a accompagné la volonté d'affermir les valeurs républicaines et de laïcité, dans un contexte où les tentations monarchistes étaient encore très vives. Comme l'écrivait, en 1929, Gustave Cauvain, directeur de l'Office régional du cinéma éducateur de Lyon: "Pour le triomphe de l'école laïque et des œuvres qui la complètent et la protègent, il est indispensable qu'une propagande intense soit faite pour et par le cinéma éducateur dans toutes les régions de notre pays $»^{13}$.

8 Ce rapprochement entre les concepts d'éducation et de propagande pose bien entendu de facto un problème relatif à la partialité inhérente à cette dernière. Emmanuel Taïeb définissait la propagande comme "un message, de forme discursive ou factuelle, mensonger ou vrai du point de vue de son émetteur, dont l'identification de la source, conjuguée à l'analyse propre du contenu, laisse supposer qu'il est partial, orienté, et qu'il n'inclut pas de contrepoint à ce qu'il contient $»^{14}$. Ainsi la propagande se caractérise-t-elle par une activité discursive qui exclut l'idée d'une pluralité de points de vue, pour ne présenter que celle à laquelle adhère son émetteur. Ce postulat est-il compatible avec les fondements même de l'éducation, dès lors qu'on la pense comme un apprentissage de la pensée critique, comme une invitation à penser par soi-même? Ceci nous ramène à l'injonction contradictoire que pointait déjà Emmanuel Kant lorsqu'il écrivait :

«Un des plus grands problèmes de l'éducation est de concilier sous une contrainte légitime la soumission avec la faculté de se servir de sa liberté. Car la contrainte est nécessaire. Mais comment cultiver la liberté par la contrainte? Il faut que j'accoutume mon élève à souffrir que sa liberté soit soumise à une contrainte, et qu'en même temps je l'instruise à en faire lui-même un bon usage. Sans cela il n'y 
aurait en lui que pur mécanisme ; l'homme privé d'éducation ne sait pas se servir de sa liberté $»^{15}$.

C'est cette contradiction inhérente à l'éducation même qui explique que l'histoire de l'éducation par l'image cinématographique - soit l'ensemble des situations où l'image cinématographique est utilisée comme support en vue de la transmission de connaissances, de concepts, de notions, de valeurs - indissociable dans l'entre-deux guerres des idéologies contraignantes qui l'ont portée, ait progressivement vu l'émergence d'un autre espace de rencontre entre l'éducation et le cinéma, à savoir l'éducation au langage cinématographique ${ }^{16}$, soit l'ensemble des actions entreprises pour permettre aux spectateurs de maîtriser les codes spécifiques au média cinématographique, dans un geste que l'on peut qualifier de sémiologique, puisqu'il s'agit en substance d'offrir les éléments d'une compréhension de la façon dont l'image animée "produit du sens $»^{17}$. Mais c'est bien à la première facette que nous nous intéressons ici, afin de comprendre comment l'histoire de la cinématographie éducative peut être comprise au prisme des idéologies qui la portent.

\section{Cinématographie éducative et propagande à l'heure de la III République}

10 Comme nous l'avons suggéré plus avant, l'histoire de la cinématographie éducative en France est tout entière traversée par l'idéologie porteuse de la III ${ }^{e}$ République, marquée par les nombreux aléas politiques qui ont fait suite à la Révolution française, soit l'avènement en moins d'un siècle deux éphémères républiques, de trois monarchies constitutionnelles et de deux empires... Les valeurs républicaines, et par extension antimonarchistes et laïques, vont constituer le ferment de de l'idéologie porteuse de la cinématographie éducative en France au cours de la période qui nous intéresse. En effet, comme le rappelait Bernard Bourdin, historien des doctrines politiques, «En France, le mot "république" a une connotation idéologique très forte : il évoque une rupture avec la référence à toute légitimation religieuse de l'institution politique $»^{18}$. L'éducation va ainsi être investie d'une mission militante, dont l'État assume pleinement la charge. Laurence Leoffel rappelait ainsi que « le principe d'une éducation libérale s'identifie ainsi à l'exigence d'une culture générale de l'esprit et à un anticléricalisme dont la dynamique est une revendication des droits de l'État à enseigner et éduquer $\aleph^{19}$. Ce sont les instituteurs - ceux-là même qui sont chargés « d'instituer » la République -, qui vont porter, pour le service de l'État éducateur, cette mission. Les idéaux révolutionnaires, eux-mêmes fondés sur l'idéologie des Lumières, ne sont pas loin, comme en témoigne par exemple le contenu du discours de Jules Ferry lors du second Congrès pédagogique des instituteurs et institutrices publics de France, le 19 avril 1881 :

« Vous êtes tous les fils de 89 ! Vous avez été affranchis comme citoyens par la Révolution française, vous allez être émancipés comme instituteurs par la République de 1880 : comment n'aimeriez-vous pas et ne feriez-vous pas aimer dans votre enseignement et la Révolution et la République ? Cette politique-là, c'est une politique nationale ; et vous pouvez, et vous devez - la chose est facile - la faire entrer, sous les formes et par les voies voulues, dans l'esprit des jeunes enfants $»^{20}$.

11 Les «hussards noirs de la République ", selon l'expression popularisée par Charles Péguy $^{21}$, se sont donc employés, par leur enseignement, à œuvrer à la consolidation de la République, de la démocratie, et cela contre, donc, la monarchie. Ce dernier point est 
important dès lors que l'Éducation nationale, telle qu'elle est pensée à l'époque, ne peut être comprise sans prendre en considération son corollaire, à savoir la lutte contre l'enseignement congréganiste délivré par l'Église, symbole de son pouvoir encore très fort sur les esprits. Pour les instituteurs, forts des leçons de Comte et de Condorcet, défendre la République supposait aussi de lutter contre l'obscurantisme, de défendre l'indépendance de la morale et de la science à l'égard de la religion. Georges Renard, dans un article daté de 1882, rappelait les fondements de ce combat:

« Ce qui fait, en effet, chez nous la plus grande force de l'Église catholique, ce n'est pas sa richesse en argent et en terres (...). C'est d'abord l'appui que lui prête l'État, la consécration officielle qu'il lui accorde généreusement, l'espèce d'auréole dont il l'entoure ainsi aux yeux des gens amoureux de l'autorité. (...). Certes, il est bien naturel que l'Église ait lutté, protesté, prêché une sorte de croisade contre les lois nouvelles. Elle s'est vue privée du double secours que lui prêtaient, pour asseoir sa domination sur les esprits, et la complicité de l'État et la mollesse des incrédules. Le prêtre et le catéchisme une fois écartés de l'école, libre sans doute aux parents qui ont la foi d'envoyer leurs enfants chercher, auprès des ministres du Culte, l'enseignement religieux ; mais libre aussi aux autres de s'en passer. (...) Et, de fait, quelle prise aura-t-elle sur des intelligences rompues, dès le jeune âge, aux habitudes scientifiques? Quel scrupule, quel respect humain pourra les arrêter dans la recherche impitoyable de la vérité ? ${ }^{22}$.

12 La mise en application de ces pratiques dans le cadre de la cinématographie éducative peut être appréhendée en trois points que nous évoquerons en répondant aux trois questions suivantes : comment l'État a-t-il accompagné la mise en place d'un cadre logistique favorable à l'usage de ces films au sein des établissements scolaires du territoire? comment ce projet a-t-il été relayé, dans le cadre péri-scolaire, par le secteur associatif? et enfin de quelle façon les films d'enseignement et d'éducation se sont chargés, à l'époque, de l'idéologie laïque et républicaine?

\section{Une « cinématographie éducative d'État » au service de la III République?}

13 La cinématographie, qui naît fort à propos en cette fin de $\mathrm{XIX}^{\mathrm{e}}$ siècle, ne va ainsi pas tarder à être mise au service de la cause républicaine. Dès les années 1910, le gouvernement de la $\mathrm{III}^{\mathrm{e}}$ République commence à réfléchir à l'opportunité d'utiliser le film en tant que support didactique et éducatif sur le territoire français. Si l'on en croit G.-Michel Coissac, dès 1912, à Bordeaux, Léon Riotor aurait évoqué, au cours du $6^{\mathrm{e}}$ congrès de la Société française de l'Art à l'école, dont il était secrétaire général, la possibilité d'organiser les usages pédagogiques du cinématographe, et appelé à l'apposition d'un agrément pour les films présentés dans les écoles, et à la création de bibliothèques de films pédagogiques ${ }^{23}$. C'est au cours de la Première Guerre mondiale que ces préoccupations reviennent à la table du gouvernement, la Commission de l'Enseignement et des Beaux-Arts à la Chambre des députés enjoignant le 23 décembre 1915 le ministre de l'Instruction publique, Paul Painlevé, à s'intéresser à la question de la cinématographie éducative ${ }^{24}$. L'objectif annoncé est de généraliser et de rationaliser l'utilisation du cinématographe dans les différentes branches de l'enseignement, sachant qu'il n'était jusque-là utilisé que de façon expérimentale par quelques enseignants assoiffés d'innovation pédagogique. Pour ce faire, une "Commission extraparlementaire du cinématographe à l'école » est créée, par décret, le 23 mars 1916. Ses travaux se poursuivent jusqu'en 1920, date à laquelle un rapport est présenté, sous 
la forme de trois points retraçant les conclusions des travaux de ses trois souscommissions :

- Les matières scolaires qui impliquent prioritairement le recours aux images animées ;

- Les qualités indispensables aux appareils de projection et aux films destinés à un usage didactique et éducatif ;

- La réalisation d'un plan d'ensemble au niveau national afin de pourvoir les écoles en matériel ciné-didactique (films, appareils de projection, écrans).

14 Ces travaux ne restèrent pas lettre morte, et l'on assista au début des années 1920 à la mise en application d'un certain nombre de propositions. Parallèlement à la mise en place d'espaces de réflexion autour des applications de la cinématographie éducative, des aides furent allouées à l'achat d'équipements cinématographiques par les établissements scolaires, une production filmique institutionnelle fut organisée, et des circuits de distribution furent spécifiquement pensés pour amener les films dans les écoles - les films résultant de la production institutionnelle bien sûr, mais aussi les films issus des catalogues des grands acteurs de la cinématographie commerciale (Pathé, Gaumont, Éclair, etc.). Ces derniers proposaient en effet nombre de films (vues, documentaires, films éducatifs...) qui présentaient un intérêt dans le cadre d'un usage didactique, si tant est qu'ils fussent en adéquation avec les besoins de l'enseignement et des valeurs de la République. L'idée d'une "liste de films agréés » vit ainsi rapidement le jour, puisque les congressistes réunis à Bordeaux en 1912 émirent le vœu que "l'introduction du cinématographe dans les écoles [soit] subordonnée aux choix des films par un conseil supérieur de l'Art à l'École ; l'usage de tout film non porté à la liste sera rigoureusement interdit; le musée de l'enseignement public sera chargé de constituer des séries qui seront mises gratuitement à la disposition des instituteurs de l'Enseignement public $»^{25}$.

Sous la supervision de l'État, plusieurs structures furent chargées de prendre en charge, de façon concrète et coordonnée, l'organisation de la cinématographie éducative sur le territoire national. On créa en premier lieu une "Cinémathèque centrale de l'Enseignement public ", au sein d'une structure préexistante, le "Musée pédagogique ». Ce dernier avait été fondé par Jules Ferry, le 13 mai 1879, et comportait une exposition permanente de matériel d'enseignement, ainsi qu'une bibliothèque. Initialement installé dans l'ancien Collège Rollin, rue Lhomond à Paris, il fut rapidement transféré, dès 1885 , dans de nouveaux bâtiments situés à l'angle de la rue Gay-Lussac et de la rue Thuillier ${ }^{26}$. Dès 1896, une nouvelle prérogative s'ajouta aux précédentes, sous la forme d'un service de prêt de vues fixes (vues sur verres pour projections lumineuses), issu d'un don conséquent en provenance de la Société nationale des Conférences populaires et de la Société d'Enseignement par les Projections Lumineuses dite "Société d'initiative pour la propagation de l'Enseignement scientifique par l'Aspect". Ce service prit rapidement une réelle ampleur, puisqu'au cours de l'année scolaire 1905-1906, plus de 32.000 boîtes avaient été expédiées vers des établissements scolaires ${ }^{27}$. C'est donc fort de cette expérience que le Musée pédagogique fut chargé, en 1920, de l'organisation d'un « Service du cinématographe à l'école ", destiné aux établissements scolaires publics, mais aussi aux conférenciers postscolaires agréés par la Direction du Musée. En 1925, le directeur du Musée pédagogique dressait le bilan suivant de l'activité cinématographique :

«Bien que ne datant que de 1920, la collection des films constitue dès maintenant un instrument fécond de développement intellectuel. Les films du ministère de l'Instruction publique, confiés au Musée pédagogique, sont exclusivement des films 
d'enseignement. L'organisation du service a été rapide, car il a bénéficié de l'expérience acquise par le personnel des vues dans le maniement, l'entretien, l'étiquetage, la réparation, l'expédition des clichés sur verre ${ }^{28}$.

Alors que seuls 54 films avaient été distribués en 1921, ils sont 29.000 en $1926^{29}$, témoignant de l'existence d'une demande réelle et en progression sur le terrain. Cette explosion de l'activité n'est pas, cependant, sans poser quelques problèmes au Musée pédagogique, problèmes qui illustrent l'insuffisance de moyens dont il a toujours souffert face à l'ampleur de la tâche qui lui était assignée: surexploitées, insuffisamment renouvelées, les copies de ses collections se dégradent rapidement, comme en témoigne en 1927 le rapport de la directrice de l'école communale de jeunes filles de l'avenue de Versailles: "Les films prêtés par le Musée pédagogique sont en mauvais état; la collection aurait besoin d'être renouvelée et surtout enrichie $»^{30}$. Par ailleurs, les enseignants se plaignent de la lourdeur du dispositif, qui les contraint à réserver plusieurs semaines à l'avance les films qu'ils souhaitent intégrer à leur enseignement, ce qui s'avère totalement incompatible avec les besoins de flexibilité qui sont les leurs, ce dont rend compte à titre d'exemple le témoignage agacé publié en 1922 dans le Bulletin de la Ligue pour l'enseignement par la cinématographie :

décembre vous parviendront en mars, lorsque votre "saison d'hiver" sera close $»^{31}$.

17 Cet engorgement explique que de nombreuses demandes restent sans suite, faute d'une capacité du Musée à y répondre, celui-ci ne parvenant " pas à satisfaire au dixième des demandes qui lui sont adressées $\|^{32}$ si l'on en croit un article publié sous la plume de Richard Avit en 1928. L'arrivée du cinéma sonore mit encore davantage en évidence le manque de moyens dont souffrait l'institution face à une demande croissante : celle-ci se montrait incapable d'intégrer à ses collections les films pédagogiques parlés qui, au grand dam de certains enseignants, révolutionnaient le rapport à l'enseignement filmé. La crise financière qui marque le début des années trente ne favorisa pas la transition, qui supposait un renouvellement du matériel de projection comme des films. C'est ce qui explique, d'ailleurs, que le cinéma soit resté «muet» dans le domaine de l'enseignement bien après que les salles se sont converties à la nouvelle industrie du cinéma sonore. Comme le remarquait un journaliste du journal Le Temps en 1935, la cinématographie scolaire semble imperméable au «progrès » : «Il existe des appareils sonores et parlants à usage scolaire, mais qui ne bénéficient et semblent ne pouvoir bénéficier d'une subvention. Celle-ci leur arrivera-t-elle un jour? $»^{33}$. Ainsi, le Musée pédagogique, tout en maintenant une réelle activité en faveur de la circulation des films éducatifs dans les écoles, souffrit d'un réel manque de moyens qui s'aggravait au fur que le temps passait. Le bilan que tirait Joseph Bréhier, ancien sénateur et président de la Ligue de l'Enseignement, devant le Sénat, lors d'une allocution datée du 19 février 1937, est particulièrement éloquent : 
«La collection du Musée Pédagogique qui comprend plus de 4.000 sujets en films muets n'est pas renouvelée en fonction de l'usure consécutive à son utilisation ; les films les meilleurs sont réduits à la moitié de leur longueur initiale et cela au détriment, évidemment, du sujet présenté. La cinémathèque des films muets du Musée pédagogique diminue en valeur tous les jours ; le musée pédagogique n'a que quelques films sonores et parlants; il ne peut en acquérir, car les crédits alloués sont nettement insuffisants ; on nous a dit à la commission du cinéma du ministère de l'Éducation nationale mardi dernier qu'il avait été dépensé en 1936, en tout et pour tout, pour les films destinés à servir à l'enseignement et à l'éducation dans toute la France, 32.000 francs ! C'est une pitié ! ${ }^{34}$.

18 D'autres institutions cependant, au cours de la même période, s'employèrent à produire, collecter, et offrir à la circulation des films pédagogiques pour les enseignants, complétant l'organisation étatique amorcée par le Musée pédagogique. Elles prirent le nom de "cinémathèques ", et ceci de façon très précoce, au début des années 1920, époque où la question de la préservation des films de fiction était encore loin d'être une préoccupation partagée ${ }^{35}$. Ces premières "cinémathèques " furent créées et gérées par les différents ministères du gouvernement français, selon des logiques thématiques liées à leurs propres préoccupations. Virent ainsi le jour des cinémathèques liées aux ministères de l'Agriculture, de la Guerre, de la Marine, du Travail, de l'Hygiène, de l'Assistance et de la Prévoyance sociale, des Travaux publics, des Affaires étrangères, des Colonies, etc. Dans chacune d'entre elles, un service de production travaillait, souvent en collaboration avec des sociétés de production privées, à la réalisation de films qui répondaient aux besoins de propagande du ministère correspondant, dans le cadre général de l'esprit de la III ${ }^{\mathrm{e}}$ République.

19 Une autre cinémathèque fut parallèlement créée par la Ville de Paris, sous l'impulsion de Léon Riotor, conseiller à la Ville de Paris depuis 1919. Ce dernier fit, dès cette annéelà, une proposition au Conseil Municipal de Paris, portée par une «Commission tendant à l'organisation de séances cinématographiques pour les enfants des écoles publiques ", qui proposait de «[doter] les écoles du matériel nécessaire aux séances, avec un opérateur ad hoc $»^{36}$. Cette proposition, qui émergeait dans le contexte de l'enthousiasme exprimé autour de la cinématographie éducative au tournant des années 1920 , se concrétisa rapidement puisque le Conseil municipal adoptait le 15 janvier 1921 le projet de création d'une Cinémathèque vouée à l'enseignement ${ }^{37}$. Celleci vit le jour quelques années plus tard, le $1^{\mathrm{er}}$ janvier 1926 , avec pour mission de « conserver des images de Paris, constituer une collection de films pour l'enseignement par achat ou par création, former les enseignants à l'utilisation des appareils de projection et à l'utilisation du film dans la classe $»^{38}$. Une activité de production propre, doublée d'une politique d'achat auprès de sociétés privées, permit la constitution progressive d'un fonds apte à soutenir les velléités ciné-éducatives des enseignants parisiens.

20 La forte centralisation dont témoignaient ces initiatives fut doublée, afin de mieux répondre aux demandes émanant des écoles partout à travers le pays, de l'organisation d'un réseau de services de distribution de films pédagogiques régionaux ou départementaux, qui permirent de prolonger à travers le territoire la mission du Musée pédagogique, asphyxié par les demandes qui lui parvenaient. Aussi, dès le début des années 1920, des structures appelées « cinémas scolaires » furent créées par plusieurs municipalités en France, soutenues en cela par le ministère de l'Instruction publique. Afin de répondre aux demandes qui dépassaient les limites de la commune, leur rayon d'action fut rapidement amené à s'étendre à un niveau régional, se transformant en 
«Office régionaux du cinéma éducateur ${ }^{39}$, abandonnant leur statut municipal pour une forme associative relevant de la loi 1901.

\section{La propagande ciné-éducative républicaine relayée par le secteur associatif}

21 La transformation des premiers "cinémas scolaires » municipaux en associations vouées à l'organisation de la cinématographie éducative dans les départements et régions françaises marqua le premier pas d'un vaste mouvement associatif en faveur de la propagande ciné-éducative républicaine. Ce témoignage, publié dans la Revue pédagogique (l'organe du Musée pédagogique) à propos de la structuration de ce réseau à Lyon puis dans toute la région lyonnaise, est symptomatique de ce changement de portée de l'action telle qu'on pouvait l'observer alors sur l'ensemble du territoire :

«Dans le courant de 1921 et 1923, le cinématographe scolaire s'est rapidement développé à Lyon. Grâce aux généreux encouragements de $M$. le Ministre, du Conseil général et de la ville de Lyon, le nombre des appareils cinématographiques s'est élevé à plus de cinquante, tant à Lyon même que dans le département. Une fois les appareils en place, toutes les difficultés n'étaient pas résolues. Il fallait: $1^{\circ}$ apprendre aux instituteurs à faire fonctionner ces appareils ; $2^{\circ}$ leur procurer à bon compte les films nécessaires qu'ils ne pouvaient trouver au dehors qu'à des prix presque prohibitifs. Dans cette intention, nous avons organisé à Lyon, dès 1922, une filmathèque qui, installée dans un local scolaire, 128, Grande-Rue de la Guillotière, est devenue de jour en jour plus riche et permet à nos instituteurs de se procurer à bas prix tous les films qui leur sont indispensables. Cette filmathèque scolaire contient à présent 750 films, se plaçant ainsi au premier rang, je crois, des filmathèques scolaires de France. Elle est surveillée et entretenue par un agent qui, moyennant une faible rétribution, apprend aux instituteurs le fonctionnement $\mathrm{du}$ cinéma et leur fait parvenir les films. Il nous a paru cette année que cette organisation était insuffisante. D'une part, les appareils deviennent de plus en plus nombreux dans le département du Rhône et les départements voisins ont commencé à nous demander de leur prêter des films. D'autre part, et par voie de conséquence, le nombre des films est destiné à s'accroître et la réparation de ces films, à leur retour dans notre filmathèque, nécessite des travaux parfois assez délicats. Nous avons donc résolu, avec l'approbation de la Municipalité de Lyon et du Conseil général, de Rhône, de fonder un Office du cinématographe scolaire dont l'activité s'étendra sur quatre départements : le Rhône, l'Isère, la Loire et l'Ain. Cet office, qui a son siège à Lyon, centralisera les fonds que lui feront parvenir les quatre départements associés et décidera de tous les achats et réparations nécessaires $»^{40}$.

Au-delà de l'extension de la portée strictement géographique de leur action, les offices régionaux du cinéma éducateur qui se créent à cette époque pensent aussi leurs activités dans un périmètre plus large : leurs services ne concernent plus exclusivement les établissements scolaires et la fourniture de films didactiques, mais s'ouvrent au champ plus vaste du « cinéma éducateur », intégrant les films de fiction dans l'arsenal ciné-éducatif, à des fins d'utilisation hors-temps scolaire, ce qui permet de combattre plus directement l'Eglise sur son propre terrain - celui de la prise en charge des enfants, hors temps scolaire, dans le cadre des patronages, au sein desquels le cinéma était utilisé tout autant pour la séduction qu'il exerçait sur les enfants que pour la propagande religieuse qu'ils permettaient de développer. En 1937, 28 offices régionaux et 40 offices départementaux étaient en exercice sur le territoire national ${ }^{41}$, regroupés depuis 1929 au sein de la Fédération nationale des offices cinématographiques 
d'enseignement, puis à partir de 1933 au sein de l'U.F.O.C.E.L. (Union française du cinéma éducateur laïque ${ }^{42}$, sous la houlette de la Ligue de l'enseignement.

Pour comprendre comment ces organismes ont naturellement constitué des relais de la propagande républicaine développée dans les écoles, par les enseignants, il est important de comprendre la double filiation idéologique qui préside à l'organisation de cette mouvance associative à travers le territoire. La première est à aller chercher du côté de l'éducation populaire: dès la fin du XIX siècle, certains conférenciers qui travaillaient dans le cadre des grandes associations d'éducation populaire qui, répondant à l'idéal révolutionnaire et républicain, se sont engagées dans la voie de l'éducation du peuple, ont commencé à intégrer des films à leur arsenal propagandistique. Dès 1899, Thomas, P.-Félix s'en extasiait dans la Revue Pédagogique: "Nos éducateurs populaires ont mis à profit dans l'intérêt de leur cause, les découvertes les plus récentes, même celle du cinématographe. Point de cours d'adultes, point de conférence sérieuse sans projections multipliées $»^{43}$. La Ligue de l'Enseignement, issue en 1866 de la volonté de républicains de permettre à tous d'accéder à l'éducation et à la culture, dans le but d'exercer pleinement leur citoyenneté et «d'établir durablement une société plus juste, plus libre, plus solidaire $\aleph^{44}$, était particulièrement active en la matière. Même si ce n'est qu'au printemps 1914 que la Ligue ouvrit officiellement un « Service des appareils et films cinématographiques ", le film avait pris place depuis de nombreuses années au sein de l'arsenal pédagogique des conférenciers.

La seconde filiation caractéristique des Offices est à chercher du côté de ce que l'on appelait alors le "cinéma ouvrier ", à savoir ce mouvement qui entendait, grâce au cinéma, insuffler l'idéal révolutionnaire - même si avant la Première Guerre mondiale, les anarchistes étaient aussi particulièrement présents sur ce terrain. Selon une logique paradoxalement très proche de celle qui guidait les instances religieuses à l'époque, l'intérêt porté au cinéma dans le mouvement ouvrier est tout d'abord lié à la volonté de lutter contre la mauvaise influence présumée exercée par les spectacles cinématographiques délivrés dans les salles d'exploitation. « Camarades, boycottons les cinés qui sabotent nos idées, obligeons-les par tous les moyens à changer leur genre de

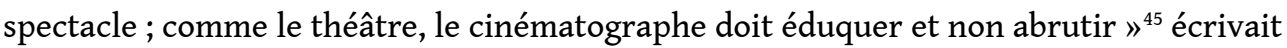
Émile Guichard dans les colonnes du Libertaire, en 1911. Parmi les actions mises en place, une société intitulée "Le Cinéma du Peuple» fut créée à Paris en 1913, se chargeant de produire et distribuer des films comme des appareils de projection, « en communion d'idées avec les groupements divers du Prolétariat ${ }^{46}$. Ainsi, quoique porteuse de convictions différentes, l'éducation ouvrière a rejoint l'éducation populaire dans certaines de ses actions. Des rapprochements se sont d'ailleurs opérés au cours de l'entre-deux-guerres entre la Ligue de l'enseignement et le mouvement ouvrier, les œuvres laïques développant une admiration certaine pour le modèle éducatif soviétique ${ }^{47}$.

Ces deux filiations se rejoignaient autour de l'objectif commun, qui a constitué la ligne directrice des Offices, consistant à lutter contre l'influence exercée sur le peuple par les patronages religieux. Ceux-ci résultaient en effet, pour reprendre les mots de Raymond Borde et Charles Perrin, de la "volonté opiniâtre de quelques militants qui se dévouaient à la République ", " combattaient l'influence de l'Église ", «militaient pour la laïcité, l'idéal coopératif, le pacifisme, l'émancipation ouvrière ${ }^{48}$. Ainsi, comme le constatait Pascal Laborderie, ces offices ont constitué, grâce au travail sans relâche 
qu'ils développaient sur le territoire tout entier, "des relais entre les services de propagande des ministères avec les divers acteurs locaux et départementaux $»^{49}$. Ils bénéficièrent d'ailleurs de mesures de soutien de la part de l'État, tout particulièrement dans les années vingt, avec en particulier la décision, en 1920, d'exonérer fiscalement les associations d'éducation populaire ${ }^{50}$.

\section{Un dispositif global efficace?}

Comme on l'a vu, les énergies sont nombreuses, diverses, et donnent lieu à d'importantes actions ciné-éducative sur l'ensemble du territoire, dans les écoles comme dans le temps hors scolaire, grâce à un partenariat public/privé naturellement permis par la rencontre autour d'un socle idéologique partagé. Cependant, le dispositif global suscita à l'époque des insatisfactions chez nombre de militants, qui espéraient une coordination plus claire de l'ensemble des initiatives ciné-éducatives. Certains souhaitaient, par exemple, que soit créé un organisme national du cinéma éducateur qui fédérerait l'ensemble des opérations sur le terrain. En 1928, Joseph Brenier, dans les colonnes du Temps, appelait de ses vœux la création d'un tel organisme, espérant qu'il puisse

«coordonner tous les efforts, toutes les initiatives qui se manifestent ou se manifesteront dans le sens de son action, comme de réduire les difficultés que l'expérience révèle tous les jours, en ce qui concerne les conditions d'attributions de subventions, l'acquisition des appareils et des films, le prêt, la circulation, le transport des bandes, l'aménagement des salles de projection, etc. $»^{51}$.

Rares sont ceux qui, comme Pierre Michaut, voyaient un avantage à l'éparpillement que connaissaient alors les réalisations en matière de cinématographie éducative. Ce dernier s'enflammait en effet, en 1935, dans les colonnes du Temps, pour vanter les bénéfices de la situation française en la matière :

«On peut donc affirmer que la légende selon laquelle le film documentaire ou scientifique français seraient en retard est fausse. Tout au contraire, nos réalisations se placent tout à fait en avant de la production mondiale. Au lieu d'être centralisées dans un Office unique, elles sont le fruit des efforts individuels de quelques dizaines de chercheurs, de savants, de curieux. Cette manifestation de notre traditionnel individualisme, loin de nuire au progrès du cinéma scolaire et scientifique français, a, au contraire, multiplié les initiatives et apporté dans les recherches une variété, une richesse, et même parfois une fantaisie qui nous valent notre incontestable avance actuelle $»^{52}$.

En réalité, les intentions qui ont accompagné le développement du cinéma éducateur en France sont globalement ternies, tout au long de l'entre-deux guerres, par l'absence d'un plan d'ensemble ambitieux, doté de financements suffisants. Au milieu des années 1930, la montée en puissance du Front populaire occasionna toutefois un nouveau regain d'intérêt gouvernemental pour la question du cinéma éducateur. Le 2 décembre 1935, on crée une "Commission du cinématographe » chargée d'étudier les questions relatives à son emploi dans les établissements d'enseignement public et dans les œuvres d'éducation postscolaire, placée sous la présidence de Paul Barrier ${ }^{53}$. Cette impulsion se poursuit avec l'arrivée au pouvoir du Front Populaire au printemps 1936, et la nomination de Jean Zay au ministère de l'Éducation nationale. En 1937, Joseph Barnier se réjouissait de cette dynamique positive dans les colonnes de la Revue du cinéma éducateur : «Depuis l'avènement du gouvernement actuel, les vœux si souvent exprimés en nos congrès de la Ligue de l'enseignement et de l'UFOCEL sont enfin 
retenus et étudiés sérieusement ; quelques-uns sont même en voie de réalisation " ${ }^{54}$. Jean Zay s'employa en particulier, par une circulaire ministérielle le 8 octobre 1935, à officialiser le $16 \mathrm{~mm}$ en tant que format réduit ciné-éducatif standard, ce qui permit par la suite la publication d'une liste des appareils agréés dans l'éducation. Un budget ministériel fut parallèlement alloué à l'achat d'appareils de projection : 390.000 francs aux communes, 322.000 francs aux œuvres postscolaires, et 70.000 francs aux cercles et patronages $^{55}$. Une nouvelle dynamique animait sans conteste les milieux politiques au regard de la cinématographie éducative, même si, comme le rappelait Pascal Ory, « il est clair qu'on a affaire ici à un cas typique d'entreprise réformatrice retardée par la "pause" ou, plus précisément, par les restrictions financières de 1937 " ${ }^{56}$.

Même si le plan d'ensemble ne fut incontestablement pas à la hauteur des espérances, l'histoire de la cinématographie éducative dans cette période constitue un exemple efficace de la mise en œuvre sur le terrain de la propagande républicaine, ce qui suppose que les films qui la portaient, dans leur structure et leurs contenus, fussent aptes à traduire les valeurs républicaines, laïques et progressistes portées par le gouvernement de la III République.

\section{Des films républicains au service de la propagande nationale}

30 Les films qui étaient utilisés dans le cadre des activités ciné-éducatives étaient majoritairement constitués de films d'enseignement et de films éducatifs, même si les films de fiction furent aussi utilisés, en particulier au sein des Offices Régionaux du cinéma éducateur. Nombre de ces films étaient produits par les grandes sociétés de production privées françaises. Ainsi, le Répertoire des films de l'encyclopédie Gaumont ${ }^{57}$, qui regroupait, par thématiques, l'ensemble des films de la firme qui pouvaient être utilisés à des fins d'enseignement, rend compte de l'intérêt évident que portaient ces sociétés de production cinématographique au marché éducatif. Le choix du terme d'encyclopédie choisi par Gaumont pour son catalogue est par ailleurs emblématique de la filiation symbolique qui s'établit en France dans l'environnement de la production cinédidactique avec l'œuvre de Diderot et D'Alembert, Elle ambitionnait, en parfaite résonnance avec l'esprit des Lumières, de rassembler puis transmettre toutes les connaissances acquises par l'humanité.

31 Nombre des films du catalogue Gaumont étaient produits en collaboration avec les ministères, et tout particulièrement avec les ministères de l'Agriculture et de l'Instruction publique, comme le précisent les génériques de ces films. L'État était ainsi très présent dans la production ciné-éducative, qui par ailleurs était plus ou moins ponctuellement prise en charge par les différents organismes que nous avons pu évoquer : l'ensemble des ministères, le Musée pédagogique, la Cinémathèque de la Ville de Paris $^{58}$, etc. Ceux qui s'en émouvaient, au nom du caractère excessivement interventionniste de l'État, étaient rares. On peut toutefois citer Émile Vuillermoz, qui s'emportait en décembre 1920 dans les colonnes du Temps:

«L'État metteur en scène, l'État scénariste, tourneur de manivelle, photographe, éditeur, exploitant et projectionniste dans toutes les écoles du territoire, voilà, certes, un cataclysme assez nettement caractérisé. Les exemples que nous connaissons de l'étatisme artistique ne nous laissent aucune illusion sur la gravité d'un tel péril. Et les quelques expériences cinématographiques tentées, çà et là, sous couleur de propagande, par tel ou tel ministère, nous ont suffisamment édifiés sur le danger de laisser des fonctionnaires jouer avec une lanterne magique $\aleph^{59}$. 
Globalement, les acteurs sur le terrain se réjouissaient de ce que les films éducatifs portent clairement l'idéologie républicaine, tendance que confirmaient d'ailleurs les films qui furent directement produits par les offices régionaux du cinéma éducateur. Plusieurs caractéristiques permettent d'énoncer un tel constat. La première est liée à la charge patriotique qui accompagnait cette production. Rappelons que l'éducation publique, telle que l'envisageait Mirabeau dès 1791, prônait la prise en charge de l'éducation par l'État en rappelant que

«c'est la plume qui conduit l'épée et qui donne ou enlève les sceptres; ce sont les instituteurs de la jeunesse, les philosophes et les écrivains de tous les genres qui font marcher les nations à la liberté, ou qui les précipitent dans l'esclavage. Il faut donc qu'ils soient toujours aux ordres de l'intérêt public $»^{60}$.

33 Ces principes furent remis au-devant de la scène à la fin du XIX ${ }^{\mathrm{e}}$ siècle, avec l'élan patriotique qui accompagna la défaite infligée par l'Allemagne en 1871 et les insurrections qui l'accompagnèrent cette année-là, dans le cadre de la Commune de Paris. Ce patriotisme marqua d'ailleurs sans conteste les milieux de l'éducation populaire. La Ligue de l'enseignement, par exemple, investira l'enseignement gymnique et militaire, créant des «bataillons scolaires " ${ }^{61}$. Raffermi par la Première Guerre mondiale, c'est naturellement que le patriotisme irrigua le cinéma éducatif dans les années vingt. Certains genres favorisèrent logiquement cette tendance. C'est le cas des films de géographie, qui exaltaient la beauté et les richesses de la France ainsi que la puissance que lui conféraient ses colonies, ou encore des films d'histoire qui entendaient raffermir le sentiment national chez les jeunes générations au contact des grandes pages de l'Histoire de France. On peut citer aussi les films scientifiques qui rendaient compte des avancées permises en la matière par les savants français.

34 Ce rapport à la science constitue le deuxième axe idéologique fort qui ancre la production ciné-didactique aux idéaux sur la base desquels s'était construite la III $^{\mathrm{e}}$ République. En effet, ces films rendent compte de la volonté de structurer la représentation du monde des spectateurs par la science, expliquant le monde par les lois scientifiques qui le régissent, rendant intelligible l'univers au gré d'une grammaire puisée dans les sciences. On y retrouve l'objectif posé par les Lumières, sur la base des travaux de Galilée et de Descartes, de "mathématiser » la structure intelligible. Bien évidemment, cet ancrage scientifique de la connaissance doit être compris comme un outil au service du combat mené contre l'influence de l'Église, de ses théories relatives au caractère divin de la création, et de ses enseignements taxés d'obscurantisme. Tous les films qui portaient sur les matières scientifiques (sciences naturelles, médecine, physique, chimie) mais aussi les films éducatifs consacrés à la prophylaxie et à l'hygiène sont alors symptomatiques de ces tendances.

35 Dans d'autres typologies génériques, cette foi en la science et les progrès qu'elle permet se traduisait par des nettes tendances à développer dans les films une vision moderniste de la société, contre les croyances rétrogrades qui enfermaient les individus dans des pratiques que l'on souhaitait proscrire, encore une fois en droite ligne avec les idéaux révolutionnaires et leurs combats contre les archaïsmes freinant les évolutions sociales. Cette tendance était très claire dans les films consacrés à l'agriculture, dont la modernisation des pratiques professionnelles (usage d'engrais, modernisation des outils, etc.) se heurtait à des croyances rétrogrades. Les films industriels, de la même façon, présentaient sous un jour très positif les évolutions technologiques et organisationnelles qui ont accompagné le début du $\mathrm{XX}^{\mathrm{e}}$ siècle. On 
peut citer aussi dans ce cadre les films qui rendaient compte des nouveaux moyens de transport et de communication qui se développaient à l'époque, et des transformations qu'ils opéraient dans la vie quotidienne.

36 La dernière caractéristique que nous évoquerons concerne l'image des filles telle qu'elle est véhiculée dans les films éducatifs. Si les femmes, privées de leurs droits civiques, sont toujours à l'époque reléguées à un rôle subalterne, la question de leur éducation constitue un rouage important de la politique de la III ${ }^{e}$ République, afin que l'éducation républicaine et l'anticléricalisme qui la conditionne soient partagés, dans les foyers, par les hommes comme par les femmes, ces dernières exerçant un pouvoir évident sur l'éducation des enfants. Ce n'est donc pas par hasard si les lois Ferry rendent obligatoire l'enseignement de 6 à 13 ans, pour les filles comme pour les garçons. L'éducation des filles constitue un motif que l'on retrouve dans les films éducatifs de l'époque. Des films d'enseignement professionnel illustraient par ailleurs le fonctionnement de filières professionnelles réservées aux jeunes filles, à l'issue du cycle d'éducation obligatoire.

37 Ainsi, à l'issue de ce rapide panorama consacré à la façon dont la cinématographie éducative a accompagné le projet politique de la $\mathrm{III}^{\mathrm{e}}$ République, on peut percevoir comment le pouvoir reconnu à l'image animée en matière de transmission de connaissances, mais aussi de valeurs et de représentations sociales, a participé de la construction d'un arsenal de propagande, porteur de convictions partagées autour des idéaux laïques et républicains. Afin d'introduire une perspective comparative dans notre réflexion, en droite ligne avec le projet de recherche à l'origine de cette publication, nous allons à présent envisager, selon les mêmes perspectives, la façon dont fut utilisée la cinématographie éducative dans le cadre politique fondamentalement différent de la période fasciste italienne.

\section{Cinématographie éducative et propagande sous le gouvernement fasciste mussolinien}

38 C'est en novembre 1922, suite à la démonstration de force opérée par les chemises noires fascistes au cours de la «Marche sur Rome» le mois précédent, que le Roi d'Italie appela Benito Mussolini à constituer un Cabinet. Ayant obtenu les pleins pouvoirs de la Chambre des députés et du Sénat, celui-ci s'employa à stabiliser son régime, renforçant rapidement la dimension totalitaire de son appareil politique, et ce jusqu'au « lois fascistissimes » votées en 1926 et 1927, qui consacraient l'organisation de l'État totalitaire. Mais avant ces lois, déjà, Mussolini ne cachait pas l'ambition qu'il poursuivait pour le pays, comme en témoigne un discours intitulé «Intransigeance absolue » qu'il tint le 22 juin 1925 devant son gouvernement :

"Ce que l'on appelle notre féroce volonté totalitaire sera poursuivie avec plus de férocité encore : elle deviendra véritablement l'obsession et la préoccupation dominante de notre activité. Nous voulons, en somme, fasciser la Nation, de sorte que demain italien et fasciste - comme pratiquement italien et catholique - seront synonymes » [Nous traduisons] ${ }^{62}$.

39 Pour « fasciser » la nation italienne, Benito Mussolini prit rapidement conscience de la nécessité de déployer une œuvre de propagande susceptible de susciter l'adhésion du peuple italien aux valeurs fascistes, et par extension à sa propre personne en tant que « Duce » (« Guide »). Le culte de la personnalité constituait en effet un élément-clé de la 
propagande telle qu'elle fut alors pensée par le régime: Mussolini était présenté comme l'homme providentiel, emblématique de "l'homme nouveau » que le fasciste était appelé à forger. On vantait sa force de caractère, son dynamisme, sa virilité, ses capacités athlétiques, autant d'attributs auxquels les Italiens étaient appelés à s'identifier. Si cette propagande eut recours à tous les dispositifs de communication alors éprouvés - les grands rassemblements populaires où il exerçait ses qualités d'orateur, mais aussi la presse, l'affichage, la radiophonie, etc. - Mussolini prit conscience, de façon tristement précoce, du rôle que pouvait jouer le cinéma dans cet arsenal de propagande. Dans son livre publié en 1932, sous le titre Le possibilità della cinematografia come mezzo di propaganda e di educazione (Les possibilités de la cinématographie comme moyen de propagande et d'éducation), Giuseppe Domenico Musso rendait compte de cette foi que l'on plaçait alors dans le cinéma :

«Le film est l'arme véritable. Cette phrase, dans sa simplicité, synthétise toute la situation. Comme la presse autrefois, la cinématographie aujourd'hui est devenue le moyen le plus puissant d'éducation et de propagande. Moyen sûr, rapide, et complètement fascinant. (...) Les avantages et la nécessité de la cinématographie puissant auxiliaire de l'enseignement, de l'éducation et de la formation de la conscience politique de nos jeunes qui un jour seront appelés à déterminer le destin de la Patrie et la défendre contre des ennemis à l'intérieur comme à l'extérieur sont tellement évidents qu'il n'est pas nécessaire de s'étendre sur la question $»^{63}$ [Nous traduisons].

Fort de ces principes, Mussolini organisa très rapidement les conditions nécessaires au déploiement d'une propagande par l'image animée à travers le pays.

\section{L'organisation par le gouvernement fasciste de la propagande filmée}

41 La première préoccupation de Mussolini consista à mettre en place les conditions d'une production ciné-éducative conforme à l'idéologie fasciste, laquelle fut prise en charge pendant toute la durée du Ventennio nero par l'Institut National LUCE. L'origine de cet organisme est à chercher dans une petite société qui avait été fondée au début de l'année $1924^{64}$ par Luciano De Feo ${ }^{65}$, sous le nom de "Sindicato Istruzione Cinematografica ", pour produire, justement, des films éducatifs. Le chef de l'État italien en eut connaissance lorsque, la même année, à l'occasion d'un voyage à Naples, il assista à la projection de quelques films produits par la petite société de production, parmi lesquels avait été introduit un court métrage consacré au quartier général du Duce, Dove si lavora per la grandezza d'Italia (Où l'on travaille pour la grandeur de l'Italie). Mussolini en fut très impressionné, et il est fort probable que cette expérience ait été déterminante dans sa perception du potentiel qu'offrait le cinéma à des fins de propagande. Peu de temps après, le Duce rebaptisa la petite société de production en lui donnant le nom de L.U.C.E, acronyme de "L'Unione Cinematografica Educativa» (L’Union Cinématographique Éducative), mais qui constitue aussi un jeu de mots avec « luce » qui signifie « lumière »). Il s'attela rapidement à mettre la société sous la coupe du régime, par le biais de son Conseil d'administration, constitué des représentants des divers organismes publics qui se répartissaient le capital par voie d'actionnariat. Giuseppe De Michelis, sénateur et commissaire général à l'Émigration, en assurait la présidence. Luciano De Feo, quant à lui, demeura à la direction du groupe. novembre 1925 un décret-loi qui transforma la société anonyme en un organisme 
parapublic : l'Institut National LUCE. Nul doute, donc, que celui-ci fût totalement au service du gouvernement fasciste, comme ses dirigeants ne manquaient pas de le rappeler dès qu'ils en avaient l'occasion. «L'Institut National LUCE a le grand honneur de dépendre directement $\mathrm{du}$ Chef $\mathrm{du}$ Gouvernement. Ce titre de noblesse, dont il est fier, lui confère l'obligation imprescriptible de se rendre digne de l'intéressement personnel du Duce, consacrant chacune de ses forces aux charges complexes et délicates qu'il doit assumer ${ }^{66}$ [Nous traduisons] pouvait-on ainsi lire dans un petit opuscule commémoratif publié en 1932. Il participa de ce fait pleinement à la « fabbrica del duce » (littéralement "l'usine du duce ») que décrit Dino Biondi dans ses travaux ${ }^{67}$, inventant les ressorts d'une véritable "industrie de la persuasion ${ }^{68}$ [Nous traduisons].

43 Cet organisme s'employa à produire l'ensemble des films nécessaires à la mise en œuvre de la propagande filmée du régime. Il bénéficia d'un monopole en la matière au niveau national à partir du 24 janvier 1929, quand fut votée la désignation de l'Institut comme unique organe technique cinématographique au service de l'État ${ }^{69}$. Par ailleurs, la question de la distribution des films avait été résolue par le gouvernement, puisqu'une loi du 3 avril 1926 obligeait les exploitants des salles de cinéma, dans tout le pays, à projeter les films que leur fournissait l'Institut National LUCE ${ }^{70}$. Et pour les zones du territoire privées de salles permanentes, un "service des projections" assurait la circulation en Italie de 25 cinémas ambulants.

Si le circuit des salles de cinéma fut très vite encadré pour permettre la circulation des films éducatifs, la question des films didactiques destinés aux établissements scolaires tarda davantage à être rationalisée. Ce n'est qu'en 1938 que le ministre de l'Éducation nationale, Giuseppe Bottai, crée une Cineteca Autonoma per la cinematografia Scolastica (Cinémathèque autonome pour la cinématographie scolaire) ${ }^{71}$, gérée par l'Institut National LUCE lui-même. Ses missions consistaient à doter les écoles du royaume en projecteurs et en films (spécialement produits à cette fin ou achetés à l'étranger), afin de parfaire dans les écoles les «objectifs politiques, éducatifs, didactiques, scientifiques, et artistiques $»^{72}$ du régime. Ceci ne signifie pas qu'une éducation par le film n'ait pas été déployée avant cette date dans les écoles du Royaume - bien au contraire, l'éducation ciné-éducative y était bien présente - mais simplement qu'aucun organisme spécifique ne la prenait en charge.

45 L'abondance de la production ciné-éducative mise en chantier par l'Institut National LUCE, les moyens dont il disposait à cet effet, la rationalité des logiques de distribution de ces films à travers le territoire n'étaient pas sans provoquer l'admiration des promoteurs de la cinématographie éducative dans le monde entier. Mussolini sut, d'ailleurs, utiliser cet avantage et la suprématie qu'il lui conférait dans ce domaine en créant à Rome, sous l'égide de la Société des Nations, un «Institut International du cinéma éducatif» qui, entre 1928 et 1936, braqua les projecteurs de l'actualité du cinéma éducatif mondial sur la capitale italienne ${ }^{73}$.

\section{La rhétorique fasciste au cœur de la production ciné-éducative}

L'Institut National LUCE offrit l'exemple d'une prise en charge centralisée, entièrement vouée à un régime totalitaire, de la production en masse de films conçus pour alimenter la propagande d'État. Cette production s'articulait autour de trois grandes typologies filmiques: les journaux cinématographiques qui étaient projetés selon un rythme hebdomadaire dans les salles de cinéma (Giornale Luce, 1928-1945); les films 
documentaires / éducatifs / didactiques, de court et long métrage (Documentari Luce, 1924-1961) ; les films de fiction, même si cette activité fut beaucoup plus réduite au cours de la période comparativement aux deux autres catégories ${ }^{74}$, qui témoignent de la façon dont, avec un peu de retard par rapport à la non-fiction, ce genre fut finalement appréhendé comme un dispositif narratif puissant pour contrôler les esprits - évolution dont témoigne par ailleurs la mise en service des studios de Cinecittà en 1937.

47 Puisque nous nous intéressons ici spécifiquement à l'usage fait de la cinématographie éducative par l'État fasciste, nous concentrerons plus précisément notre propos sur les " Documentari ", même si en réalité les trois genres participaient du même élan que la propagande fasciste exerçait sur les esprits. La production de ces films s'articulait au sein de l'Institut National LUCE autour des différentes "cinémathèques » (cineteche) thématiques qui furent successivement créées : la Cinémathèque agricole nationale (18 mars 1926) ; la Cinémathèque industrielle ( 6 août 1926) ; la Cinémathèque d'art et d'Instruction religieuse (21 octobre 1926) ; la Cinémathèque de culture nationale (3 novembre 1926) ; la Cinémathèque militaire d'instruction et de propagande (30 janvier 1927) ; la Cinémathèque touristique et de propagande maritime (30 janvier 1927) ; la Cinémathèque d'hygiène et de prévention sociale (30 janvier 1927) ; la Cinémathèque pour la propagande et la culture à l'étranger (2 juin 1927) ${ }^{75}$. Chacune de ces branches articulait, dans la sphère qui était la sienne, l'élaboration du projet, la réalisation des films, leur diffusion, mais aussi la conservation des négatifs et des copies de projection.

L'intitulé de chacune de ces sections montre parfaitement comment la production était rationalisée autour des grandes œuvres du régime - bien loin de la perspective encyclopédique que nous avons relevé dans le catalogue Gaumont. Il s'agissait en effet d'afficher la production ciné-éducative comme pensée au gré d'une interaction permanente avec les orientations adoptées par le régime fasciste. Nous parlons volontairement d'affichage ici, dans la mesure où, en réalité, les films produits par l'Institut National LUCE relevaient de catégories assez semblables à ce que l'on pouvait observer en France: on y trouvait des films de géographie, d'histoire, des films scientifiques (par ailleurs de grande qualité) ${ }^{76}$, des films agricoles, des films ethnologiques, des films folkloriques, des films d'art, etc. Chacune de ces typologies de production, toutefois, était traversée par une vision du monde en parfaite adéquation avec la pensée fasciste, au fur et à mesure de ses formulations successives au cours du Ventennio. C'est le cas, par exemple, des nombreux films de géographie, qui constituèrent au cours des années trente le réceptacle des velléités colonialistes et guerrières qui ont accompagné la campagne d'Abyssinie. Notons toutefois que la vision coloniale, et le racisme qui la conditionne, imprégnait de la même façon les films de géographie français de la même époque, mais la nécessité de faire adhérer le peuple italien aux efforts de guerre, dans le contexte d'une idéologie vantant la supériorité de l'italianité sur les autres peuples, a engendré une exacerbation nauséabonde du nationalisme sur lequel se fondait l'idéologie fasciste mussolinienne. Au-delà de la propagande réalisée sur le territoire italien, les films éducatifs furent d'ailleurs aussi utilisés sur le terrain de guerre, et considérés comme des "instruments de pénétration ${ }^{77}$ particulièrement efficaces. En témoigne ce reportage publié par le journal français Le Temps, qui rend compte par ailleurs du regard particulièrement complaisant que les démocraties ont jeté sur le régime fasciste italien et ses manifestations, et cela jusqu'à la condamnation de l'Italie par la Société des Nations 
consécutive à l'annexion de l'Éthiopie et la signature de l'Axe Rome-Berlin, en 1935 et 1936 :

« Le cinéma va jouer son rôle dans la pénétration italienne au Tigré. Aujourd'hui mardi, pour la première fois, les gens d'Adoua verront se projeter des images mouvantes sur un écran. La projection se fera en plein air, sur la principale place d'Adoua. Le général Maravigna, M. Bottai, gouverneur de Rome, les officiers et soldats de la garnison y assisteront. On s'attend que tous les indigènes de la ville et des environs soient attirés par ce spectacle, insolite pour eux. Ils verront des films sur de grandes manifestations patriotiques ou leur montrant la puissance militaire italienne, notamment des documentaires sur les manœuvres navales, et sur les Grandes manouvres de Bolzano. Ces films seront commentés dans la langue du pays $»^{78}$.

49 Au-delà de la question militaire, tous les grands événements ou orientations qui marquaient la politique du régime trouvaient leur traduction dans les films éducatifs de la LUCE : la «bataille du blé » et la «bonifica agraria » (politique d'assèchement des marais pontins) qui caractérisèrent la politique agraire furent largement documentées ; la politique nataliste irriguait l'ensemble des représentations de la famille; les grands travaux entrepris pour lutter contre le chômage (monuments, stades, autoroutes, etc.) et la reconstruction industrielle donnaient lieu à de nombreux films exaltant les œuvres du régime, etc. L'exaltation de la force physique donnait lieu à des films hygiénistes, vantant les activités physiques, ou montrant le Duce lui-même s'adonnant à des sports variés. De nombreux films s'employaient ainsi à démontrer les bienfaits du sport, appelé à "régénérer » la race italienne dont la "pureté de sang séculaire est le plus grand titre de noblesse de la nation italienne ${ }^{79}$ [Nous traduisons]. Les sports qui supposaient de l'audace et du courage, comme l'aviation, l'alpinisme, étaient naturellement privilégiés dans la production ciné-éducative, afin de renforcer les aspirations héroïques des Italiens. La production ciné-éducative fut ainsi tout entière conçue comme un outil au service de l'œuvre de propagande nationale. « En relisant les catalogues des films de la cinémathèque Luce, nous voyons à quel point l'œuvre éducative du Gouvernement National a été étendue à tous les champs de la laborieuse activité italienne pour le renouveau moral et physique de la race $»^{80}$ [Nous traduisons] peut-on lire en 1933 sous la plume d'Iperato Zanetti.

\section{Les espaces de circulation des films éducatifs}

50 La propagande par le film telle que l'imaginait Mussolini s'adressait à tous les Italiens, quels que soient leur âge et leur condition sociale. D'où le recours aux salles de cinéma $\mathrm{du}$ Royaume, qui accueillaient les populations friandes de divertissements cinématographiques, assurant ainsi aux films de l'Institut National LUCE un public varié, abondant, et captif puisque le visionnage des films de fiction était conditionné par celui des pré-programmes éducatifs.

51 La question se posait toutefois dans des termes quel que peu différents pour ce qui concernait le jeune public, pour lequel une médiation humaine s'avérait utile pour accompagner le viosionnage des films et assurer la formation des jeunes esprits à l'idéologie du régime. C'est pourquoi ce dispositif de base fut complété par des propositions annexes.

52 Tout d'abord, l'école fut investie en tant qu'espace privilégié d'accès aux jeunes générations, en particulier après le tournant fascisant dont fut l'objet le système 
scolaire italien après 1925, au moment où Giovanni Gentile fut remplacé au poste de ministre de l'Instruction publique par Pietro Fedele, lequel s'attela à mettre en œuvre les ambitions mussoliniennes concernant l'institution scolaire: "Le gouvernement exige que toute école, à tous les degrés, dans tous les enseignements, éduque la jeunesse dans l'intelligence du fascisme, lui enseigne à trouver sa noblesse dans le fascisme, à vivre dans le climat historique issu de la révolution fasciste $»^{81}$ [Nous traduisons] déclarait Benito Mussolini le 5 décembre 1925 devant la Corporation de l'École. Le projet consistant à utiliser l'école en tant qu'espace privilégié pour modeler les esprits enfantins, réprimer toute vision du monde autre que celle proposée par le fascisme, était ainsi clairement énoncée, et c'est dans ce cadre que les films éducatifs furent utilisés au sein des établissements scolaires du Royaume pendant la période.

Parallèlement à l'école, les organismes créés par le fascisme pour enrégimenter les jeunes générations accueillirent aussi les films éducatifs en tant qu'outils de propagande. Nous faisons allusion ici à l'œeuvre Nationale Balilla (ONB), une société morale chargée de l'assistance et de l'éducation physique et morale de la jeunesse des deux sexes de moins de dix-huit $a^{82}{ }^{82}$, au gré de sections spécialisées pour les différentes classes d'âge ${ }^{83}$. Cette organisation bénéficiait d'un monopole en matière d'encadrement de la jeunesse dans le pays, assurant ainsi l'unilatéralité de la vision du monde proposée aux jeunes générations. L'intégration des jeunes fut rendue obligatoire à partir de 1930, les enseignants étant chargés de systématiquement inscrire les élèves dans les œuvres de l'ONB, les deux organes travaillant de concert aux mêmes objectifs :

«Vous devez vous rappeler que le but visé par l'€uvre Nationale Balilla est précisément celui auquel tend l'école : construire une civilisation italienne qui, partant de l'élévation de l'esprit jointe à la vigueur du corps, suscite et développe toutes les énergies propres à la race, les rattache aux fins de la grandeur de la Patrie et conserve aux Italiens la physionomie particulière qui les caractérise, même parmi les peuples les plus évolués, comme les héritiers d'une civilisation supérieure $\aleph^{84}$.

54 Cet embrigadement conjoint de la jeunesse connut son apogée en 1935 lorsque, dans le contexte d'une survalorisation des ambitions impérialistes du régime, la préparation militaire de la jeunesse devint un mot d'ordre partagé, afin de « forger une race saine, disciplinée, virile, rompue à tous les exercices physiques et prête à tous les sacrifices $»^{85}$.

Dans le contexte de l'école comme de l'ONB, les films produit par l'Institut National LUCE - puis plus tard par l'Incom -furent utilisés au gré d'objectifs partagés, que retraçaient parfaitement les déclarations d'intention qui ont accompagné la création de la Cineteca Autonoma per la cinematografia Scolastica au sein de l'Institut National LUCE: les films étaient appelés à revêtir une "physionomie italienne " ${ }^{86}$, afin que "l'école politique puisse finalement détruire les séquelles de l'instruction publique et faire de l'enseignement et du savoir un idéal, un objectif formaliste $»^{87}$ [Nous traduisons]. On prôna aussi, tout particulièrement dans la dernière période du fascisme, l'utilisation au sein de l'école de films proposant une autoreprésentation de l'institution scolaire fasciste. On insistait aussi sur la possibilité qu'offraient de tels films de stimuler l'esprit de compétition des élèves - envisagé comme ferment du nationalisme - en illustrant par le film des compétitions sportives scolaires, ou des manifestations militaires auxquelles auraient participé certaines classes. 
On vantait aussi le recours, en classe, aux films portant sur l'histoire de l'art, auxquels on attribuait des qualités particulières en matière d'enrégimentement des esprits, du fait même de la grandeur de l'histoire artistique italienne qui constituait un vecteur idéal des valeurs nationalistes du régime: "L'utilisation des images animées dans l'école permet à l'élève (...) de s'abandonner à la suggestion d'œuvres d'art vivantes et de pressentir ainsi ces idéaux de vie et ces problèmes idéologiques qui un jour imprégneront son esprit et sa conscience d'homme $»^{88}$ [Nous traduisons] pouvait-on lire sous la plume de Mario Alfredo Alla en 1938. Ces films permettaient entre autres de faire l'apologie du «surhomme » - thème omniprésent dans la rhétorique fasciste, en particulier dans les représentations faites du Duce - les artistes étant survalorisés en raison de leur force de travail incomparable. La représentation des corps modelés dans les œuvres classiques et néo-classiques s'accompagnait d'une exaltation de la magnificence du corps sain, puissant, vigoureux - qui préfigurait l'armée puissante et résistante célébrée par la rhétorique guerrière du régime ${ }^{89}$.

L'œuvre de propagande ciné-éducative du régime fasciste s'est ainsi développée au cours du Ventennio au sein de dispositifs variés, et dans le contexte de thématiques extrêmement diverses mais qui, toutes, convergeaient vers un même objectif: représenter le monde environnant, les connaissances, l'histoire de l'Humanité au prisme de représentations convergentes conformes au strict carcan idéologique élaboré par le régime fasciste mussolinien.

\section{Conclusion}

58 L'observation des grandes lignes autour desquelles se sont développées les cinématographies éducatives en France et en Italie au cours de l'entre-deux guerre met en évidence l'existence d'une intentionnalité partagée, consistant à mettre ce nouvel outil, venu s'ajouter à la palette des «imageries éducatives » en vigueur, au service d'un récit auquel l'État éducateur souhaitait voir adhérer le plus grand nombre. Dans les deux cas, ce récit est envisagé comme un "récit-monde», offrant une vision cohérente de l'ordre social, au gré d'une structure idéologique partagée par les émetteurs de ces messages. Sa transmission est assurée à différents niveaux, de façon systématique et totalement encadrée dans le cas de l'Italie fasciste, en s'appuyant sur des relais plus diversifiés - le secteur associatif en particulier - dans le cas de la France. Dans le cadre autoritaire du régime fasciste, aucun espace contradictoire n'est toléré, et la cinématographie éducative se limite à cet environnement communicationnel encadré par l'État : celui-ci élabore les messages, soit en les produisant lui-même, soit en contrôlant très scrupuleusement ceux qui auraient été produits à l'étranger, et veille à leur ample circulation jusqu'à leurs destinataires. En France, ce récit subit les assauts d'un opposant identifié - en tant qu'actant dans le modèle proposé par Greimas opposant qui utilisait les mêmes ressorts de propagande par le film pour assoir sa propre vision du monde. L'Église catholique, en effet, comprit très tôt l'usage qu'elle pouvait faire du film en tant qu'outil éducatif, et utilisa les mêmes armes cinééducatives pour rallier à sa cause, les enfants dans les patronages, et les adultes dans les cinémas paroissials qu'elle gérait sur le territoire.

59 En fin de compte, si les idéologies qui animaient la III ${ }^{\mathrm{e}}$ République française et l'État fasciste italien étaient diamétralement opposées, leurs usages du cinéma éducatif comme outil en faveur de leur propre propagande répondent à des intentions assez 
similaires. Et si l'on se défend en France de procéder, à l'image des dictatures voisines, $\mathrm{au}$ "dressage » du peuple ${ }^{90}$, si les promoteurs de la cinématographie éducative ancrent leur discours dans un ensemble de valeurs héritées des Lumières pour se démarquer de l'endoctrinement auquel se livrent leurs voisins européens, leur action ciné-éducative reste, comme toute œuvre éducative, profondément enracinée dans un terreau idéologique partagé. En 1934, Émile Roux-Parassac, personnalité parmi les plus actives en faveur de la cinématographie éducative, écrivait :

« Pour nous, le cinéma éducateur, le vrai, plane au-dessus des contingences ; il n'est pas motif ou prétexte à favoriser telle idée, telle opinion particulière pour contrecarrer d'autres idées et opinions de même bonne ou mauvaise foi, si respectables que puissent être les unes et les autres ; mais un guide indépendant, le guide tout court, capable d'apprendre à discerner, à juger, à décider, à devenir meilleur. (...) À notre humble avis, en matière d'éducation, le film ne saurait être une arme de combat, mais un généreux et digne livre de raison $»^{91}$.

60 Joseph Brenier, Président de la Ligue de l'enseignement, allait dans le même sens lorsqu'en 1937, il s'inquiétait devant le Sénat des dérives de l'usage du film éducatif :

« En Italie, en Allemagne, en Russie, le cinéma et la radio tiennent une place de plus en plus grande dans la propagande en faveur du régime, mais aussi dans la formation culturelle des adolescents et des enfants. Un simple chiffre : l'Allemagne a équipé à l'heure actuelle plus de 60.000 de ses écoles, et les appareils, qui sont en général des $16 \mathrm{~mm}$, servent aussi dans les réunions de propagande ; tous les films, récréatifs et scolaires, se terminent par l'apologie de Hitler ; il en est de même en Italie pour Mussolini (...) En France, il ne peut être question d'utiliser le cinéma éducateur à quelque propagande politique que ce soit; il doit œuvrer dans le respect des principes qui ont présidé à l'organisation et au fonctionnement de notre école publique $»^{92}$.

61 Si Joseph Brenier tend, dans son discours, à distinguer clairement les usages français de l'éducation par le film de ceux qui se développent chez ses voisins européens, la crispation progressive mais inéluctable des relations internationales ouvre la voie à des aspirations plus décisives dont Guy Laborde rendait compte lorsqu'il écrivait dans les colonnes du Temps :

" Au moment où un peuple voisin fort de plus de 60 millions d'habitants proclame sa foi mystique en une supériorité de race qui le placerait au-dessus et en dehors de la communauté humaine, lorsqu'il met aux mains d'une jeunesse fanatisée par ses éducateurs un armement gigantesque, les maîtres français trahiraient leur fonction si, gardant le silence, ils n'employaient pas l'autorité dont ils jouissent pour éclairer notre peuple abusé si longtemps $»^{93}$.

62 Ainsi, dans ce contexte, la fin des années trente va être fortement marquée par cette prise de conscience de la puissance du cinéma en tant qu'outil de propagande, et du danger qu'il représente lorsqu'il est manié par des régimes totalitaires. Si le constat était évident au regard du cinéma éducatif, le cinéma de fiction commençait lui aussi à être observé avec méfiance. En résulte une défiance globale à l'encontre de l'image animée, que l'on perçoit de moins en moins comme le "miroir du monde ", et de plus en plus comme porteuse d'une idéologie nécessitant un décryptage de la part du récepteur, une connaissance des mécanismes de son langage propre. Les réflexions qui en découlent constituent les racines d'une nouvelle préoccupation qui va petit à petit se construire dans l'environnement propre à la rencontre entre le cinéma et l'éducation: la nécessité de développer une «éducation au langage cinématographique ", indispensable à la conservation d'une autonomie de la pensée critique et d'une résistance à l'endoctrinement. Mais ceci est une autre histoire... 


\section{BIBLIOGRAPHIE}

Alla, Mario Alfredo. « Le nuove forze - Il cinéma », in La scuola fascista, Tivoli, collezione di studi della rivista "La Terra", vol. III, 1938, p. 64.

Argentieri, Mino. L'occhio del regime. Informazione e propaganda nel cinema del fascismo. Florence, Vallecchi editore, 1979.

Augé, Étienne F. Petit traité de propagande à l'usage de ceux qui la subissent. Bruxelles, De Boeck, 2007. Auzias, Jean-Marie. L'anthropologie contemporaine : expérience et système. Paris, PUF, 1976.

Avit, Richard. « Le cinéma post-scolaire. Conditions de son plus grand développement », Le travail manuel, les sciences expérimentales, et le cinéma à l'école, $\mathrm{n}^{\circ} 9,1^{\mathrm{er}}$ juin 1928, p. 289.

Berstein, Serge ; Milza, Pierre. L'Italie contemporaine des nationalistes aux Européens. Paris, Armand Colin, 1973.

Bessou, Auguste. Commission extraparlementaire chargée d'étudier les moyens de généraliser l'application du cinématographe dans les différentes branches de l'enseignement : rapport général / présenté par M. Aug. Bessou, Paris, ministère de l'Instruction publique et des Beaux-Arts, Imprimerie nationale, 1920.

Betti, Carmen. L'Opera Nazionale Balilla e l'educazione fascista. Florence, La nuova Italia Editrice, 1984.

Biondi, Dino. La fabbrica del duce. Florence, Vallecchi, 1967.

Borde, Raymond ; Perrin, Charles. Les offices du cinéma éducateur et la survivance du Muet (1925-1940). Lyon, Presses universitaires de Lyon, 1992.

Branca, Remo. Il cinema nella scuola, Novara, Regio Istituto Magistrale 'Contessa Giuseppa Tornielli Bellini', 1939.

Brunetta, Gian Piero ; Dottorini, Daniele. Enciclopedia del cinema. Trecani, 2003.

Catalogue de la société Gaumont : Société des établissements Gaumont. Répertoire des films de l'encyclopédie Gaumont, Paris, Société des établissements Gaumont, Service de l'enseignement, s.d. (env. 1921).

Cauvain, Gustave. Persévérer, Rapport sur l'activité et le développement de l'office Régional du Cinéma Éducateur de Lyon en 1928. Lyon, Office Régional du cinéma éducateur, 1929.

Coissac, G.-Michel. Le cinématographe et l'enseignement. Nouveau Guide pratique. Paris, Édition du Cinéopse, 1926.

De Pastre-Robert, Béatrice. « Enfer, amnésie, rédemption : une histoire du fonds de la cinémathèque scolaire Robert-Lynen », in De Pastre, Béatrice ; Dubost, Monique ; Massit-Folléa, Françoise (dir.), Cinéma pédagogique et scientifique. À la redécouverte des archives. Paris, ENS éditions, 2004, pp. 45-58.

Devos, Emmanuelle. La cinémathèque de la Ville de Paris. Les idées et les faits (1906-1938), Université de la Sorbonne Nouvelle Paris III, Mémoire de maîtrise en études cinématographiques (sous la direction de Michel Marie), 1996.

Domenach, Élise. « Le cinéma exprime-t-il le scepticisme », in Cerisuelo, Marc ; Laugier, Sandra. Staney Cavell : cinéma et philosophie. Paris, Presses de la Sorbonne Nouvelle, 2001, pp. 215-238. 
Ferry, Jules. « Discours au second Congrès pédagogique des instituteurs et institutrices publics de France, 19 avril 1881 », Cité dans : Ferry, Jules ; Robiquet, Paul. Discours et Opinions de Jules Ferry, Tome quatrième, Les lois Scolaires (suite et fin). Paris, Armand Colin, 1896.

Gauthier, Christophe. «Au risque du spectacle. Les projections cinématographiques en milieu scolaire dans les années 1920 ", in De Pastre, Béatrice ; Dubost, Monique ; Massit-Folléa, Françoise (dir.), Cinéma pédagogique et scientifique. À la redécouverte des archives, Paris, ENS Éditions, 2004, pp. 73-98.

Gillette, Aaron. «The Origins of the "Manifesto of Racial Scientists" », Journal of Modern Italian Studies, $n^{\circ}$ 6, 2001, pp. 305-323.

Guillemoteau, René. Du Musée pédagogique à l'Institut pédagogique national. 1879-1956. Paris, Centre national de documentation pédagogique, 1979.

Jaspers, Karl. Origine et sens de l'histoire. Paris, Plon, 1954.

Kant, Emmanuel. Traité de pédagogie (traduction Jules Barni). Paris, Félix Alcan éditeur, 1886.

Laborderie, Pascal. Le film-parabole dans les offices du "cinéma éducateur" en France dans l'entre-deuxguerres. Histoire d'un cinéma de propagande et étude d'un genre cinématographique. Thèse en études cinématographiques et audiovisuelles, Université Paris 3-Sorbonne Nouvelle, 2009.

Laura, Ernesto G. Le stagioni dell'aquila. Storia dell'Istituto Luce. Rome, Ente dello Spettacolo editore, 2000.

Lecompte, Jean-Michel ; Sylvestre, Jean-Pierre (dir.). Culture républicaine, citoyenneté et lien social. Éditions CNDP, SRDP de Bourgogne, Documents, actes et rapports pour l'éducation, 1997.

Léglise, Paul. Histoire de la politique du cinéma français. T.1 : Le cinéma et la troisième République. Paris, Pierre Lherminier éditeur, 1977.

Lenoir, Yves ; Vanhulle, Sabine. «Les finalités en éducation : des discours socio-idéologiques aux positionnements épistémologiques et axiologiques ", in Favre, Daniel et al., Les valeurs explicites et implicites dans la formation des enseignants, Louvain-la-Neuve [Belgique], De Boeck Supérieur, 2008, pp. 55-72.

Loeffel, Laurence. « Aux sources de l'éducation laïque et libérale : spiritualisme et libéralisme en France au XIX"e siècle ", Les Sciences de l'éducation - Pour l'Ère nouvelle, 2008/2, Vol. 41, pp. 25-43.

Manetti, Daniela. "Un'arma poderosissima". Industria cinematografica e Stato durante il fascismo 1922-1943. Milan, Franco Angeli Editore, 2012.

Mannoni, Laurent. « 28 octobre 1913 : création de la société "Le cinéma du peuple" », 1895, Mille huit cent quatre-vingt-quinze, Paris, hors-série L'année 1913 en France, octobre 1993, pp. 100-107.

Martel, Angéline ; Villeneuve, Daniel. « Idéologies de la nation, idéologies de l'éducation au Canada entre 1867 et 1960 : le "bénéfice du locuteur" majoritaire ou minoritaire ", Revue canadienne de l'éducation, 20/3, 1995, pp. 392-406.

Michaut, Pierre. « Le cinéma scientifique et éducateur en France. Troisième congrès de l'Association pour la documentation cinématographique dans les sciences », Le Temps, Paris, n²7.074, 19 octobre 1935 , p. 5.

Mirabeau (comte de. - De Riquetti Honoré-Gabriel), « Discours sur l'éducation nationale », in Mirabeau (comte de. - De Riquetti Honoré-Gabriel) ; Barthe, Félix. Discours et opinions de Mirabeau ; précédés d'une notice historique sur sa vie. Vol. 3. Kleffer et A. Caunes, 1820. 
Musée pédagogique, service central des projections lumineuses (cinématographe), ministère de l'Éducation nationale, Centre national de documentation pédagogique, n4, 1933.

Musso, Giuseppe Domenico. Le possibilità della cinematografia come mezzo di propaganda e di educazione. Rome, Luzzetti, 1932.

Mussolini, Benito. Scritti e discorsi dal 1925 al 1926, vol. V. Milan, Ulrico Hoepli, 1934.

Niney, François. L'épreuve du réel à l'écran. Essai sur le principe de réalité documentaire. Bruxelles, Éditions De Boeck, 2002.

Odin, Roger. Cinéma et production de sens. Paris, Armand Colin, 1990.

Odin, Roger. De la fiction, Bruxelles, De Boeck, 2000.

Origine, organizzazione e attività dell'Istituto Nazionale LUCE. Rome, Istituto Poligrafico dello Stato, 1934.

Ory, Pascal. La politique culturelle du Front populaire français : 1935-1938. Thèse pour le doctorat d'État en Histoire, Université Paris Ouest Nanterre La Défense, 1990.

Peguy, Charles. «L'Argent », in Cahiers de la Quinzaine, Paris, 9e et 6e cahiers de la 14e série, 1913.

Pietrafesa, Maria. Guida agli archivi della cineteca Lucana. Censimento degli archivi privati conservati dall'Associazione Cineteca Lucana di Oppido Lucano. Soprintendenza archivistica per la Basilicata, octobre 2012.

Rébillon, L. « Une coopérative de films d'enseignement », Ciné-Schola. Bulletin de la Ligue pour l'enseignement par la cinématographie, ${ }^{\circ} 2$, décembre 1922, pp. 6-8.

Renaitour, Jean-Michel. Où va le cinéma français ? Paris, Éditions Baudinière, 1937.

Renard, Georges. « La Réforme de l'instruction populaire en France et ses conséquences », in La Nouvelle Revue, Paris, novembre 1882, pp. 495-497.

Riotor, Léon. Conseil Municipal de Paris. Rapports et documents. Imprimerie municipale, Hôtel de Ville, n¹14, 27 décembre 1919.

Schaff, Adam. «La définition fonctionnelle de l'idéologie et le problème de la "fin du siècle de l'idéologie" ", L’Homme et la société, n 4, 1967, pp. 49-59.

Sévilla, Nathalie. La Ligue de l'enseignement-Confédération générale des œuvres laïques 1919-1939. Thèse de doctorat d'histoire (direction : Jean-François Sirinelli), Institut d'études politiques de Paris, 2004, 2 vol.

Taïeb, Emmanuel. «La propagande revisitée », Quaderni [en ligne], n72, Printemps 2010, pp. 5-18. Taillibert, Christel. L'Institut international du cinématographe éducatif. Regards sur le rôle du cinéma éducatif dans la politique internationale du fascisme italien. Paris, L'Harmattan, 1999.

Taillibert, Christel ; D’Arcangeli, Marco Antonio. « Luciano De Feo: un'internazionalista pacifista nell'Italia di Mussolini? », Cinema e Storia, Anno V, n²1, 2017, pp. 35-50.

Thomas, P.-Félix. « L’enseignement visuel », in Revue pédagogique, Paris, n¹2, 15 décembre 1899, p. 484.

Tournemire, Pierre. La Ligue de l'enseignement, Toulouse, Édition Milan, 2000.

Van Dijk, Teun. « Politique, Idéologie et Discours », Semen, n²1, 2006 [en ligne] mis en ligne le 16 janvier $2007 \mathrm{http}: / /$ semen.revues.org/1970

Vuillermoz, Émile. «Strangulation », Le Temps, Paris, n²1685, 15 décembre 1920, p. 3. 
Zanetti, Iperato. La cinematografia e la scuola fascista. La cinematografia maestra di vita. Triestre, s.e., 1933.

\section{NOTES}

1. Martel, Angéline ; Villeneuve, Daniel. «Idéologies de la nation, idéologies de l'éducation au Canada entre 1867 et 1960 : le "bénéfice du locuteur" majoritaire ou minoritaire ", Revue canadienne de l'éducation, 20/3, 1995, p. 392.

2. Lenoir, Yves ; Vanhulle, Sabine. "Les finalités en éducation : des discours socio-idéologiques aux positionnements épistémologiques et axiologiques ", in Favre, Daniel et al., Les valeurs explicites et implicites dans la formation des enseignants, Louvain-la-Neuve [Belgique], De Boeck Supérieur, 2008, p. 57.

3. Ibidem.

4. Auzias, Jean-Marie. L'anthropologie contemporaine: expérience et système. Paris, PUF, 1976, p. 22.

5. Selon le modèle sémio-pragmatique proposé par Roger Odin, les «modes " correspondent à une "construction théorique visant à structurer en ensembles fonctionnels les processus de production de sens » (Odin, Roger. De la fiction, Bruxelles, De Boeck, 2000, p. 46).

6. Domenach, Élise. "Le cinéma exprime-t-il le scepticisme", in Cerisuelo, Marc; Laugier, Sandra. Staney Cavell : cinéma et philosophie. Paris, Presses de la Sorbonne Nouvelle, 2001, p. 234.

7. Niney, François. L'épreuve du réel à l'écran. Essai sur le principe de réalité documentaire. Bruxelles, Éditions De Boeck, 2002, p. 71.

8. Van Dijk, Teun. «Politique, Idéologie et Discours », Semen, n²1, 2006 [en ligne] mis en ligne le 16 janvier 2007 http://semen.revues.org/1970

9. Schaff, Adam. «La définition fonctionnelle de l'idéologie et le problème de la "fin du siècle de l'idéologie" ", L’Homme et la société, n 4, 1967, p. 50.

10. Une acception péjorative du terme a été très largement partagée en Sciences sociales, en raison de la popularité de la conception exprimée par le philosophe Karl Jaspers, pour qui : «Une idéologie est un complexe d'idées ou de représentations qui passe aux yeux du sujet pour une interprétation du monde ou de sa propre situation, qui lui représente la vérité absolue, mais sous la forme d'une illusion par quoi il se justifie, dissimule, se dérobe d'une façon ou l'une autre, mais pour son avantage immédiat. Voir qu'une pensée est idéologique équivaut à dévoiler l'erreur, à démasquer le mal, la designer comme idéologie, c'est lui reprocher d'être mensongère et malhonnête, on ne saurait donc l'attaquer plus violemment » (Jaspers, Karl. Origine et sens de l'histoire. Paris, Plon, 1954, p. 403).

11. Le terme « idéologie » vient du grec, : idea (idée) et logos (science).

12. Augé, Étienne F. Petit traité de propagande à l'usage de ceux qui la subissent. Bruxelles, De Boeck, 2007, p. 12.

13. Cauvain, Gustave. Persévérer, Rapport sur l'activité et le développement de l'Office Régional du Cinéma Éducateur de Lyon en 1928. Lyon, Office Régional du cinéma éducateur, 1929, p. 41.

14. Taïeb, Emmanuel. « La propagande revisitée », Quaderni [en ligne], n72, Printemps 2010, p. 10. Mis en ligne le 05 avril 2012 http://quaderni.revues.org/475

15. Kant, Emmanuel. Traité de pédagogie (traduction Jules Barni). Paris, Félix Alcan éditeur, 1886, p. 57.

16. Un troisième espace dans la rencontre entre ces deux concepts que sont le cinéma et l'éducation peut être désigné en tant « qu'éducation à la culture cinématographique ", entendue comme l'ensemble des situations où le cinéma devient l'objet même du dispositif éducatif : 
l'histoire de ses courants esthétiques, de ses œuvres marquantes, de son environnement technologique, de ses évolutions socio-économiques, des hommes et femmes qui ont accompagné son évolution, des politiques culturelles et législatives, etc. deviennent ainsi les éléments d'une culture à partager. Ces trois espaces sont communément regroupés dans ce que l'on désigne aujourd'hui en France sous l'expression « éducation à l'image ».

17. L'expression est ici empruntée à Roger Odin, dans l'ouvrage : Cinéma et production de sens. Paris, Armand Colin, 1990.

18. Bourdin, Bernard. "La République ou la frontière du visible », in Lecompte, Jean-Michel ; Sylvestre, Jean-Pierre (dir.). Culture républicaine, citoyenneté et lien social. Éditions CNDP, SRDP de Bourgogne, Documents, actes et rapports pour l'éducation, 1997, p. 9.

19. Loeffel, Laurence. « Aux sources de l'éducation laïque et libérale : spiritualisme et libéralisme en France au XIX siècle », Les Sciences de l'éducation - Pour l'Ère nouvelle, 2008/2, Vol. 41, p. 26.

20. Ferry, Jules. «Discours au second Congrès pédagogique des instituteurs et institutrices publics de France, 19 avril 1881 », Cité dans : Ferry, Jules ; Robiquet, Paul. Discours et Opinions de Jules Ferry, Tome quatrième, Les lois Scolaires (suite et fin). Paris, Armand Colin, 1896.

21. Péguy, Charles. "L'Argent ", in Cahiers de la Quinzaine, Paris, 9e et 6e cahiers de la 14e série, 1913 [en ligne] site du CNDP, consulté le 15 mai 2014 http://www2.cndp.fr/ revuedar/pdf/75503562/20722611.pdf

22. Renard, Georges. "La Réforme de l'instruction populaire en France et ses conséquences ", in La Nouvelle Revue, Paris, novembre 1882, pp. 495-497.

23. Coissac, G.-Michel. Le cinématographe et l'enseignement. Nouveau Guide pratique. Paris, Édition du Cinéopse, 1926, p. 38.

24. Rapporté dans: Bessou, Auguste. Commission extraparlementaire chargée d'étudier les moyens de généraliser l'application du cinématographe dans les différentes branches de l'enseignement : rapport général / présenté par M. Aug. Bessou, Paris, ministère de l'Instruction publique et des Beaux-Arts, Imprimerie nationale, 1920, p. 1.

25. Texte repris dans : "Rapport et document du Conseil Municipal de la Ville de Paris ", ${ }^{\circ} 42$, 1926, p. 3. Document cité dans : Devos, Emmanuelle. La cinémathèque de la Ville de Paris. Les idées et les faits (1906-1938), Université de la Sorbonne Nouvelle Paris III, Mémoire de maîtrise en études cinématographiques (sous la direction de Michel Marie), 1996, pp. 9-10.

26. Guillemoteau, René. Du Musée pédagogique à l'Institut pédagogique national. 1879-1956. Paris, Centre national de documentation pédagogique, 1979, pp. 11-12.

27. Ibid, p. 44.

28. Ibid., p. 69.

29. Musée pédagogique, service central des projections lumineuses (cinématographe), ministère de l'Éducation nationale, Centre national de documentation pédagogique, ${ }^{\circ} 4,1933$, p. 2.

30. Rapport de la directrice de l'école communale de jeunes filles de l'avenue de Versailles, 29 juillet 1927, 2 f., Archives de Paris, D2T1. Cité par Gauthier, Christophe. «Au risque du spectacle. Les projections cinématographiques en milieu scolaire dans les années 1920 ", in De Pastre, Béatrice ; Dubost, Monique ; Massit-Folléa, Françoise (dir.), Cinéma pédagogique et scientifique. À la redécouverte des archives, Paris, ENS Éditions, 2004, p. 87.

31. Rébillon, L. «Une coopérative de films d'enseignement », Ciné-Schola. Bulletin de la Ligue pour l'enseignement par la cinématographie, $n^{\circ} 2$, décembre 1922, p. 7.

32. Avit, Richard. «Le cinéma post-scolaire. Conditions de son plus grand développement ", Le travail manuel, les sciences expérimentales, et le cinéma à l'école, $\mathrm{n}^{\circ} 9,1^{\mathrm{er}}$ juin 1928, p. 289.

33. «Le Cinéma scolaire et le progrès », Le Temps, Paris, 3 novembre 1935, n 27.089, p. 4.

34. Cité dans: Renaitour, Jean-Michel. Où va le cinéma français? Paris, Éditions Baudinière, 1937, pp. 193-194. 
35. La Cinémathèque française ne fut créée qu'en 1936, sous une forme associative et sur la base d'une initiative privée.

36. Riotor, Léon. Conseil Municipal de Paris. Rapports et documents. Imprimerie municipale, Hôtel de Ville, $\mathrm{n}^{\circ} 114,27$ décembre 1919.

37. Rapporté dans : «L'éducation scolaire par le cinématographe ", in L'Art à l'École. Bulletin de la Société française, Paris, juin 1921, n³, pp. 69-71.

38. De Pastre-Robert, Béatrice. «Enfer, amnésie, rédemption: une histoire du fonds de la cinémathèque scolaire Robert-Lynen », in De Pastre, Béatrice ; Dubost, Monique ; Massit-Folléa, Françoise (dir.), Cinéma pédagogique et scientifique. À la redécouverte des archives. Paris, ENS éditions, 2004, p. 47.

39. On pourra se référer, à propos de cette histoire singulière, aux travaux de Pascal Laborderie y compris à l'article qu'il publie dans le présent volume - ainsi qu'aux travaux précurseurs de Raymond Borde et Charles Perrin: Les offices du cinéma éducateur et la survivance du Muet (1925-1940). Lyon, Presses universitaires de Lyon, 1992.

40. " Filmathèque scolaire ", in Revue pédagogique, Paris, $\mathrm{n}^{\circ} 6$, juin 1924, pp. 363-364.

41. Brenier, Joseph. Sixième séance, tenue au Sénat, le 19 février 1937, retranscrite dans : Renaitour, Jean-Michel. Op. Cit., p. 209.

42. Tournemire, Pierre. La Ligue de l'enseignement, Toulouse, Édition Milan, 2000, p. 24.

43. Thomas, P.-Félix. «L'enseignement visuel ", in Revue pédagogique, Paris, $\mathrm{n}^{\circ} 12,15$ décembre 1899, p. 484.

44. Tournemire, Pierre. Op. Cit., p. 3.

45. «Le ciné peut être moralisateur, il peut avoir une influence considérable sur l'éducation », in Le Libertaire, n³1, 13 mai 1911. Cité dans Mannoni, Laurent. « 28 octobre 1913 : création de la société "Le cinéma du peuple" », 1895, Mille huit cent quatre-vingt-quinze, Paris, hors-série L'année 1913 en France, octobre 1993, p. 103.

46. Acte de fondation de «Le Cinéma du Peuple », 28 octobre 1913 (Ibid., p. 104).

47. Sévilla, Nathalie. La Ligue de l'enseignement-Confédération générale des œuvres laïques 1919-1939. Thèse de doctorat d'histoire (direction : Jean-François Sirinelli), Institut d'études politiques de Paris, 2004, 2 vol., chap. IX.

48. Borde, Raymond ; Perrin, Charles. Op. Cit., pp. 7-8.

49. Laborderie, Pascal. Le film-parabole dans les offices du "cinéma éducateur" en France dans l'entredeux-guerres. Histoire d'un cinéma de propagande et étude d'un genre cinématographique. Thèse en études cinématographiques et audiovisuelles, Université Paris 3-Sorbonne Nouvelle, 2009, p. 53.

50. Léglise, Paul. Histoire de la politique du cinéma français. T.1: Le cinéma et la troisième République. Paris, Pierre Lherminier éditeur, 1977.

51. «L'organisation du cinéma d'enseignement », Le Temps, Paris, 23 août 1928, n²4476, p. 3.

52. Michaut, Pierre. «Le cinéma scientifique et éducateur en France. Troisième congrès de l'Association pour la documentation cinématographique dans les sciences ", Le Temps, Paris, n²7.074, 19 octobre 1935 , p. 5.

53. Ses trois sous-commissions étudièrent respectivement les questions techniques (soit l'agrément des appareils susceptibles d'être utilisés dans l'enseignement public), les questions pédagogiques (soit l'examen des films existant, et à l'élaboration programmatique de films à réaliser) et enfin les questions commerciales et douanières.

54. Brenier, Joseph. Revue du cinéma éducateur, janvier 1937, cité dans: Ory, Pascal. La politique culturelle du Front populaire français: 1935-1938. Thèse pour le doctorat d'État en Histoire, Université Paris Ouest Nanterre La Défense, 1990, vol. 3, p. 1256.

55. Ibid., p. 1257.

56. Ibid., p. 1256. 
57. Catalogue de la société Gaumont: Société des établissements Gaumont. Répertoire des films de l'encyclopédie Gaumont, Paris, Société des établissements Gaumont, Service de l'enseignement, s.d. (env. 1921).

58. Musée pédagogique, service central des projections lumineuses (cinématographe), Op. Cit., p.4.

59. Vuillermoz, Émile. «Strangulation », Le Temps, Paris, n²1685, 15 décembre 1920, p. 3.

60. Mirabeau (comte de. - De Riquetti Honoré-Gabriel), « Discours sur l'éducation nationale », in Mirabeau (comte de. - De Riquetti Honoré-Gabriel) ; Barthe, Félix. Discours et opinions de Mirabeau ; précédés d'une notice historique sur sa vie. Vol. 3. Kleffer et A. Caunes, 1820, p. 511.

61. Tournemire, Pierre. Op. Cit., p. 7.

62. Mussolini, Benito. «Intransigenza assoluta », 22 juin 1925, in Scritti e discorsi dal 1925 al 1926, vol. V. Milan, Ulrico Hoepli, 1934, p. 109.

63. Musso, Giuseppe Domenico. Le possibilità della cinematografia come mezzo di propaganda e di educazione. Rome, Luzzetti, 1932, p. 19.

64. Dans son ouvrage sur l'histoire de l'Institut National LUCE, Ernesto G. Laura rend compte des incertitudes quant à la date exacte de création de cette société, mais plusieurs sources convergent autour du début de l'année 1924. (Laura, Ernesto G. Le stagioni dell'aquila. Storia dell'Istituto Luce. Rome, Ente dello Spettacolo editore, 2000, p. 23).

65. À propos de cette personnalité très importante dans l'histoire de la cinématographie éducative en Italie, on pourra se référer à l'article suivant: Taillibert, Christel ; D'Arcangeli, Marco Antonio. « Luciano De Feo: un'internazionalista pacifista nell'Italia di Mussolini? », Cinema e Storia, Anno V, n¹, 2017, pp. 35-50.

66. Origine, organizzazione e attività dell'Istituto Nazionale LUCE. Rome, Istituto Poligrafico dello Stato, 1934, p. 2.

67. Biondi, Dino. La fabbrica del duce. Florence, Vallecchi, 1967.

68. Traduction de "industria della persuasione ", expression utilisée par Mino Argentieri dans son ouvrage : L'occhio del regime. Informazione e propaganda nel cinema del fascismo. Florence, Vallecchi editore, 1979, p. 9.

69. Cette situation de monopole prit fin en 1938, lorsque le gouvernement fasciste-créa un autre organisme, qui s'installa dans les tous nouveaux bâtiments de Cinecittà : l'Incom (Industria Corto Metraggi). Celui-ci se spécialisa dans la production de courts métrages documentaires d'auteur, dont l'Institut National LUCE assurait la distribution.

70. Regge Decreto-Legge du 3 avril 1926, n¹000. «Provvedimenti per la propaganda a mezzo della cinematografia, in Lex, I. pp. 1414-1415. Cité dans: Manetti, Daniela. "Un'arma poderosissima". Industria cinematografica e Stato durante il fascismo 1922-1943. Milan, Franco Angeli Editore, 2012, p. 54.

71. Cette cinémathèque survécut à la chute du régime fasciste, et poursuivit son activité jusqu'au début des années cinquante. Ses archives sont aujourd'hui conservées par la Cineteca Lucana, et sont constituées d'environ 7.000 copies de courts métrages didactiques italiens et étrangers, 20.000 films fixes, 6.000 ouvrages, des diapositives touristiques, 500 appareils de projection et les archives liées à l'activité de cette institution (Pietrafesa, Maria. Guida agli archivi della cineteca Lucana. Censimento degli archivi privati conservati dall'Associazione Cineteca Lucana di Oppido Lucano. Soprintendenza archivistica per la Basilicata, octobre 2012, p. 15).

72. Branca, Remo. Il cinema nella scuola, Novara, Regio Istituto Magistrale 'Contessa Giuseppa Tornielli Bellini', 1939, p. 6.

73. Voir à ce propos : Taillibert, Christel. L'Institut international du cinématographe éducatif. Regards sur le rôle du cinéma éducatif dans la politique internationale du fascisme italien. Paris, L'Harmattan, 1999. Cet ouvrage est disponible en langue italienne: L'Istituto Internazionale per la cinematografia educativa. Il ruolo del cinema educativo nella 
politica internazionale del fascismo italiano [traduction de Marco Antonio D'Arcangeli]. Rome, Anicia, collana Insegnare il Novecento, 2019.

74. On peut citer, parmi les films les plus marquants: Camicia nera (Giovacchino Forzano, 1933) ; Il cammino degli eroi (Corrado D'Errico, 1936) Scipione l'Africano (Carmine Gallone, 1937) ; Grano fra due battaglie (Romolo Marcellini, 1941) ; I trecento della settima (Mario Baffico, 1943), etc. Voir : Brunetta, Gian Piero. «Istituto Nazionale Luce » in Brunetta, Gian Piero ; Dottorini, Daniele. Enciclopedia del cinema. Trecani, 2003.

75. Laura, Ernesto G. Op. Cit., pp. 31-34.

76. L'Institut National LUCE s'est très vite équipé de laboratoires extrêmement modernes en vue de produire des films scientifiques. La direction de cette section a été confiée en 1926 au célèbre Roberto Omegna, qui s'était fait connaître dès le début du siècle pour ses documentaires réalisés au sein de la société Ambrosio Film.

77. «Le Cinéma, instrument de pénétration », Le temps, 23 octobre 1935, p. 2.

78. Ibidem.

79. «Manifesto degli scienziati razzisti », 1938, reproduit dans : Gillette, Aaron. «The Origins of the "Manifesto of Racial Scientists" ", Journal of Modern Italian Studies, n 6, 2001, p. 319.

80. Zanetti, Iperato. La cinematografia e la scuola fascista. La cinematografia maestra di vita. Triestre, s.e., 1933, p 3.

81. Discours devant la Corporation de l'Ecole du 5 décembre 1925 retracé dans: Mussolini, Benito. Op. Cit., p. 218.

82. Betti, Carmen. L'Opera Nazionale Balilla e l'educazione fascista. Florence, La nuova Italia Editrice, 1984.

83. Dès 6 ans, les enfants étaient appelés à intégrer les «Figli della lupa " (enfants de la louve). Les garçons, à 8 ans, devenaient «Balilla», puis «Balilla Moschettiere» à 13 ans, « Avanguardita » à 14 ans, « Avangardista Moschettiere » à 17 ans, «Giovane fascista » ̀̀ 21 ans. Les filles, quant à elles, devenaient « Piccola italiana » à 8 ans, puis « Giovane italiana » à 15 ans, et ce jusqu’à leur majorité

84. Cité dans : Bulletin du Bureau international d'éducation, Genève, $\mathrm{n}^{\circ} 15$, avril 1930.

85. Berstein, Serge; Milza, Pierre. L'Italie contemporaine des nationalistes aux Européens. Paris, Armand Colin, 1973, p. 276.

86. Branca, Remo. Op. Cit., p 11.

87. Ibidem.

88. Alla, Mario Alfredo. «Le nuove forze - Il cinéma », in La scuola fascista, Tivoli, collezione di studi della rivista "La Terra", vol. III, 1938, p. 64.

89. On pourra se référer à propos de la charge idéologique de ces films à l'article suivant: Taillibert, Christel. «Les films sur l'art produits en Italie au cours de la période fasciste », in Valentine Robert, Laurent Le Forestier, François Albéra (dir.), Le film sur l'art - Entre histoire de l'art et documentaire de création, Presses universitaires de Rennes, collection « Le Spectaculaire », 2015, pp. 55-68.

90. «Ils [les maîtres] ne songent certes pas à imiter leurs collègues allemands et à transformer l'enseignement en une propagande. L'école française n'a pas besoin de ces grossiers procédés de dressage » pouvait-on lire en 1936 sous la plume de Guy Laborde (Laborde, Guy. «La Ligue de l'éducation française ", Le Temps, Paris, n²7.232, 26 mars 1936, p. 8).

91. Roux-Parassac, Émile. "Du cinéma éducateur ", in La participation française au congrès d'enseignement et d'éducation. Paris, Comité français de l'Institut international du cinématographe éducatif, 1934, p. CLXXXV.

92. Brenier, Joseph. Sixième séance, tenue au Sénat, le 19 février 1937, retranscrite dans : Renaitour, Jean-Michel. Op. Cit.., p. 199. 


\section{RÉSUMÉS}

L'image animée a constitué au début du $\mathrm{XX}^{\mathrm{e}}$ siècle un nouvel outil au service de la propagande éducative des États désireux de transmettre aux populations une vision du monde, un récit partagé, un socle identitaire commun. En abordant successivement, dans une perspective comparative, les situations propres à la $\mathrm{III}^{\mathrm{e}}$ République française et à la période fasciste mussolinienne, cet article se propose d'analyser la façon dont la cinématographie éducative a été pensée puis organisée par les deux États, et comment les films utilisés se sont chargés de leurs idéologies respectives.

\section{INDEX}

Mots-clés : Cinéma éducatif ; propagande ; idéologie ; IIIe République ; fascisme italien ; Mussolini

\section{AUTEUR}

\section{CHRISTEL TAILLIBERT}

Christel Taillibert est maître de conférences HDR en Sciences de l'Information et de la communication au sein de l'Université Côte d'Azur. Ses recherches se développent au sein du LIRCES (Laboratoire Interdisciplinaire Récits, Cultures et Sociétés) et portent principalement sur les médiations cinéma / éducation : histoire de la cinématographie éducative, histoire de la cinéphilie, éducation à l'image, festivals de cinéma, plateformes cinéphiles, etc. Ses travaux récents interrogent d'une part les problématiques liées à la mise à disposition des images animées dans les environnements numériques (éditorialisation des contenus / usages), et d'autre part les pratiques de réemploi autour des archives de cinéma amateur. 